Timon Rabczuk • Stéphane Bordas •

Goangseup Zi

\title{
A three dimensional meshfree method for continuous multiple-crack initiation, propagation and junction in statics and dynamics
}

Received: date / Accepted: date

\begin{abstract}
This paper proposes a three dimensional meshfree method for arbitrary crack initiation and propagation that ensures crack path continuity for non-linear material models and cohesive laws. The method is based on a local partition of unity. An extrinsic enrichment of the meshfree shape functions is used with discontinuous and near-front branch functions to close the crack front and improve accuracy. The crack is hereby modelled as a jump in the displacement field. The initiation and propagation of a crack is determined by the loss of hyperbolicity or the loss of material stability criterion. The method is applied to several static, quasi-static and dynamic crack problems. The numerical results very precisely replicate available experimental and analytical results.
\end{abstract}

Keywords extended element free Galerkin method (XEFG); three dimensional cracks; cohesive forces; static and dynamic fracture; extrinsic partition of unity enrichment; non-linear fracture mechanics.

Timon Rabczuk

Institute for Numerical Mechanics, Technical University of Munich, Boltzmannstr. 15, D-85748 Garching b. Munich, Germany

Tel.: +49-89-289915304

E-mail: Timon.Rabczuk@lnm.mw.tum.de

Stéphane Bordas

Ecole Polytechnique Fédérale de Lausanne (EPFL) Laboratoire de structures et de mécanique des milieux continus, Station 18, CH-1015 Lausanne, Switzerland Tel. +41-21-693-24-03

E-mail: stephane.bordas@alumni.northwestern.edu

Goangseup Zi

Department of Civil and Environmental Engineering, Korea University, 5-1, Anamdong, Sungbuk- Ku, Seoul 136-701, Korea

E-mail: g-zi@korea.ac.kr 


\section{INTRODUCTION}

Three dimensional modelling of crack initiation and propagation still poses essential difficulties, especially when crack path continuity should be enforced. In two dimensions, the crack is simply a line and propagation is just the extension of this line. In three dimensions, we are dealing with a crack surface that can be concave or convex. The crack surface can be represented by segments or by other methods such as level sets. It is still a difficult task to propagate the crack front in three dimensional space and many assumptions have to be made. Another difficulty is to model crack junctions if crack path continuity should be guaranteed. In computational fracture dynamics, for instance, the crack path tends to look rather erratic if no smoothing procedure is used. In finite elements, patch recovery techniques of Zienkiewicz and Zhu type [56] is typically employed. Although the smoother shape functions ${ }^{1}$ available to meshfree methods meet less difficulties, a smoothing of the crack normal guarantees that the kinks in the crack path are less severe. If such severe kinks remain, it is very difficult to maintain crack path continuity without considering the joining of cracks in the background cells.

Crack branching that often occurs under dynamic loading conditions, Liu et al. [31], is another challenging point in fracture mechanics, especially in $3 \mathrm{D}$, and only few results are available.

There are very few approaches so far in the literature that can handle arbitrary crack propagation in three dimensions and preserve crack path continuity at the same time. One of the first methods that can handle arbitrary crack propagation in three dimensions was developed by Martha et al. [36] in a finite element context. Many methods are restricted to planar crack growth and linear elastic fracture mechanics (LEFM) or were applied only for such cases. The methods of Xu and Ortiz [53], Xu et al. [52] and Galdos [20], for instance, are models that can handle planar three dimensional cracks for elastostatics.

Cervenka [13] proposed a method to model cracks in three dimensional solids based on a fracture mechanics concept. For linear elastic fracture mechanics, Moes et al. [38] and Gravouil et al. [22] published two subsequent papers how to treat arbitrary non-planar three dimensional cracks in the extended finite element method (XFEM). The crack surface was represented smoothly and traced with level sets. They showed for several examples impressive results. Bordas [10] used XFEM for damage tolerance analysis of complex industrial structures by three dimensional crack propagation in the LEFM context as well. Lo et al. [34]; Chopp and Sukumar [15] presented interesting methods for multiple planar cracks in three dimension for LEFM.

Areias and Belytschko [3] employed XFEM to model three dimensional cohesive crack initiation and propagation for non-linear materials. The crack was represented by triangles. A similar approach was pursued by Gasser and Holzapfel [21] within the partition of unity method (PUFEM). Though reasonable results were presented, they did not enforce crack path continuity in general but across certain lines. Simkins and $\mathrm{Li}$ [48] proposed a very interesting technique to describe the crack surface using the parametric visibility

\footnotetext{
${ }^{1}$ higher order of continuity
} 
criterion [30] and a splitting particle technique. In their method, no particles have to be added around the crack tip such that "remeshing" and the associated mapping from the old to the new mesh can be avoided. Recently, Oliver et al. [42] compared embedded elements and the XFEM for three dimensional crack problems, which showed that embedded elements can give results very comparable to the XFEM.

The only articles (according to the knowledge of the authors) that describe the application of the element-free Galerkin method to three dimensional crack problems are the papers by Krysl and Belytschko [27]; Sukumar et al. [49] and Duflot [19]. In all three papers, the visibility or the diffraction method was used to trace the crack. While the two latter papers deal with LEFM cracks in statics, the first paper by Krysl and Belytschko [27] proposes a general method in elastodynamics.

We propose a simple method to model three dimensional crack initiation and crack growth in meshfree methods when crack path continuity is desired. Loss of hyperbolicity or Rankine criterion is chosen as transition criteria to the discontinuity. The direction of the crack is given by a discontinuous bifurcation analysis and completely arbitrary. When a crack advances, i.e. when the crack front is updated, it is restricted to advance through an entire background integration cell. The update of the crack front at each time step is therefore governed by the size of the background integration cells ahead of the crack front. The piecewise-continuous crack front is comprised of line segments that always lie on the edges of the tetrahedral background cells. From the above, we see that the crack interior is represented by piecewise continuous planar segments, each of which is always a cross section of one of the tetrahedral background cells. To help guarantee crack path continuity of a propagating crack front, we smooth the crack normal obtained from the localization analysis.

When a Gauß point loses material stability, a crack initiates (and propagates). The radius of the penny-shaped crack initiation is governed by the domain of influence of the node that is the closest to the Gauß point. The crack is introduced through all the background cells that are covered by the domain of influence of that node. We use a near-front enrichment function to ensure crack closure at the crack front and use adaptivity to improve the accuracy around the crack front. Though we do not need to remesh, a certain refinement around the crack tip is needed to get adequate results. This was already noted by Bordas [10]; Bordas and Moran [11] and Gasser and Holzapfel [21] who modelled fracture of concrete in three dimensions. Therefore, we use $h$-adaptivity and add nodes around the crack tip. The use of $h p$-adaptivity would also be possible. Applications of $h p$-adaptivity and practical implementations of $h p$-adaptive techniques can be found in Monk [39]; L. and Demkovicz [28]; Demkovicz and Vardapetyan [17]; Rachowicz and Demkovicz [46, 47].

We have developed a technique for meshfree methods which enables us to initiate and propagate cracks arbitrarily through three-dimensional space, based on material stability criteria and treat crack junction of not only two previously initiated cracks but also two "simultaneously" initiating cracks. Our method is different from the above mentioned methods in several ways. 
First, most methods described above do not consider crack initiation but only the propagation of a pre-given crack. For instance, in finite elements, it is very difficult or impossible to close the crack front within a single element, see Bellec and Dolbow [6]. In contrast to most other methods mentioned above, we use a crack tip enrichment to close the crack at the crack front. This is a by-product of meshfree methods due to strongly overlapping domains of shape functions and has the advantage that accuracy can be significantly improved for certain classes of problems, e.g. in LEFM. The tip enrichment vanishes on the crack front and thus enables the crack to close exactly along the crack front. The minimal "radius" ${ }^{2}$ of a newly initiating crack is the size of the domain of influence of the closest particle to the crack surface.

Second, as hinted at above, our technique treats crack junctions in three dimensions within a single background cell, which is an essential feature of the method, making it flexible, versatile, and robust.

Third, we use adaptivity for a better accuracy around the crack tip. Adaptivity is also advantageous for crack initiation, since the crack radius of newly initiated cracks is significantly smaller, and to enforce crack path continuity since a better resolution of the crack front can be achieved.

We would also like to mention that the crack propagation algorithm is closely related to the cracking criteria used. We use loss of material stability criterion where the orientation of the crack is uniquely determined by the bifurcation analysis. With this method, it can happen that severe changes in the crack orientation occur along a crack front, thereby requiring a very fine mesh to ensure complete crack path continuity. The aforementioned methods do not treat this case naturally. One type of methods described above does not enforce crack path continuity completely, e.g. only at certain lines that can lead to locking and a mesh orientation bias. The other type of methods are able to enforce crack path continuity completely but they have to ignore the normal obtained from the bifurcation analysis, since a triangular plane segment is uniquely determined once two of its edges are defined. In this case, the order in which cracking happens becomes also important. Again, this is problematic for curved crack fronts where the crack orientation differs severely. We overcome this difficulty with adaptivity that provides a fine resolution around the crack tip and by smoothing the crack normals. In the examples we have shown here, this was sufficient. However, we have also tested examples with highly curved cracks where we encountered difficulties. We have found that different crack segments maintain the correct normal depending on the order in which background cells crack. With increasing mesh refinement, these difficulties are alleviated.

The paper is arranged as follows: we first describe the crack kinematics and the approximation of the displacement field. In the following section, we

\footnotetext{
${ }^{2}$ A crack is described by a set of contiguous piecewise linear plane segments composed of the sections of tetrahedral background cells with the cracking plane. Moreover, the crack front is a set of piecewise linear line segments in three dimensions, composed of the trace of this plane on the faces of the background cells. Consequently, a "penny" crack is only an approximation of a disk, whose accuracy is dictated by the refinement of the refinement of the background integration mesh. This characteristic can be seen in Figure 7, and is revisited in the body of the article.
} 
give the governing equations and derive the discrete equations. We briefly review the constitutive models used for the uncracked continuum part and explain crack initiation/propagation criteria, the cohesive law and some implementational aspects. We finally apply the method to several static and dynamic examples that are compared to experimental data or other results from the literature, and close with some conclusions and future work options.

\section{APPROXIMATION OF THE DISPLACEMENT FIELD}

In the remainder of the paper, a node or particle is a point carrying the primary field variables. We will use the term localization analysis and discontinuous bifurcation analysis interchangeably. The term background cell refers to the integration cells used for integration of the weak form. In the implementation, we chose to put a node on all vertices of all background cells, but this is by no means a requirement. Shape functions are built by the Moving Least Squares method (MLS), and a cubic B-spline is used as a weight function.

The basic idea is the decomposition of the displacement field into a continuous part $\hat{\mathbf{u}}$ and discontinuous part $\tilde{\mathbf{u}}$ :

$\mathbf{u}=\hat{\mathbf{u}}+\tilde{\mathbf{u}}$

$\hat{\mathbf{u}}(\mathbf{X})=\sum_{I \in \mathcal{W}(\mathbf{X})} \Phi_{I}(\mathbf{X}) \mathbf{u}_{I}$

$\tilde{\mathbf{u}}(\mathbf{X})=\sum_{I \in \mathcal{W}_{b}(\mathbf{X})} \Phi_{I}(\mathbf{X}) \bar{H}\left(f_{I}(\mathbf{X})\right) \mathbf{a}_{I}+\sum_{I \in \mathcal{W}_{s}(\mathbf{X})} \Phi_{I}(\mathbf{X}) \sum_{K} \overline{\mathbf{B}}_{K}(\mathbf{X}) \mathbf{b}_{K I}$

with

$\bar{H}\left(f_{I}(\mathbf{X})\right)=H(f(\mathbf{X}))-H\left(f\left(\mathbf{X}_{I}\right)\right)$

$\overline{\mathbf{B}}_{K}(\mathbf{X})=\mathbf{B}(\mathbf{X})-\mathbf{B}\left(\mathbf{X}_{K}\right)$

where $\Phi_{I}(\mathbf{X})$ are the shape functions, $H$ and $\mathbf{B}$ are the enrichment functions explained later, $f$ is the signed distance function, $\mathcal{W}(\mathbf{X})$ are all the nodes in the entire domain and $\mathcal{W}_{b}(\mathbf{X}), \mathcal{W}_{s}(\mathbf{X})$ are the nodes whose domains of influence are completely and partially cut by a crack as shown in figure 1, respectively. We use the element-free Galerkin method with linear complete shape functions; for further details see e.g. Rabczuk et al. [45]; Belytschko et al. [8]; Liu et al. [33, 32]. The test functions have the same structure.

$\hat{\mathbf{u}}$ in eq. (2) is the usual approximation, the first and the second terms on the RHS of Equation (3) are the enrichment. The array of coefficients a and $\mathbf{b}$, are additional unknowns introduced to represent the discontinuity across the crack faces and the asymptotic stress state approaching the crack tip, respectively. $H\left(f\left(\mathbf{X}_{I}\right)\right)$ depends on the signed distance function $f\left(\mathbf{X}_{I}\right)$ and is defined as:

$\begin{array}{ll}H\left(f\left(\mathbf{X}_{I}\right)\right)=1 & \text { if } f\left(\mathbf{X}_{I}\right)>0 \\ H\left(f\left(\mathbf{X}_{I}\right)\right)=-1 & \text { if } f\left(\mathbf{X}_{I}\right)<0\end{array}$ 


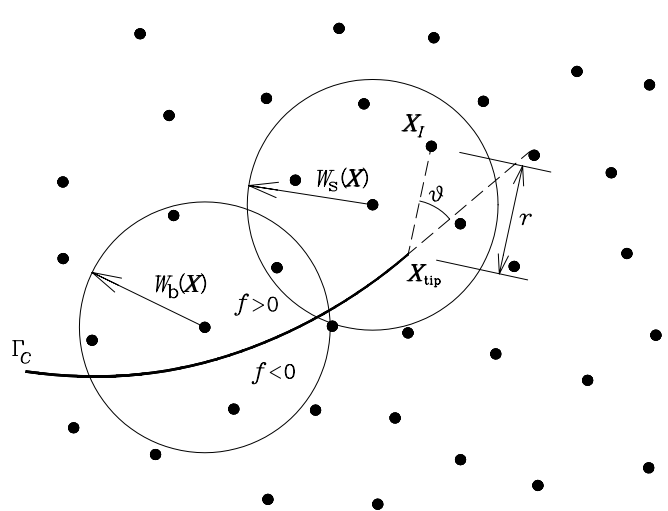

(a) Definition of the crack tip coordinate system for the two dimensional case. Particles' domains of influence may be partially or completely cut by the crack.

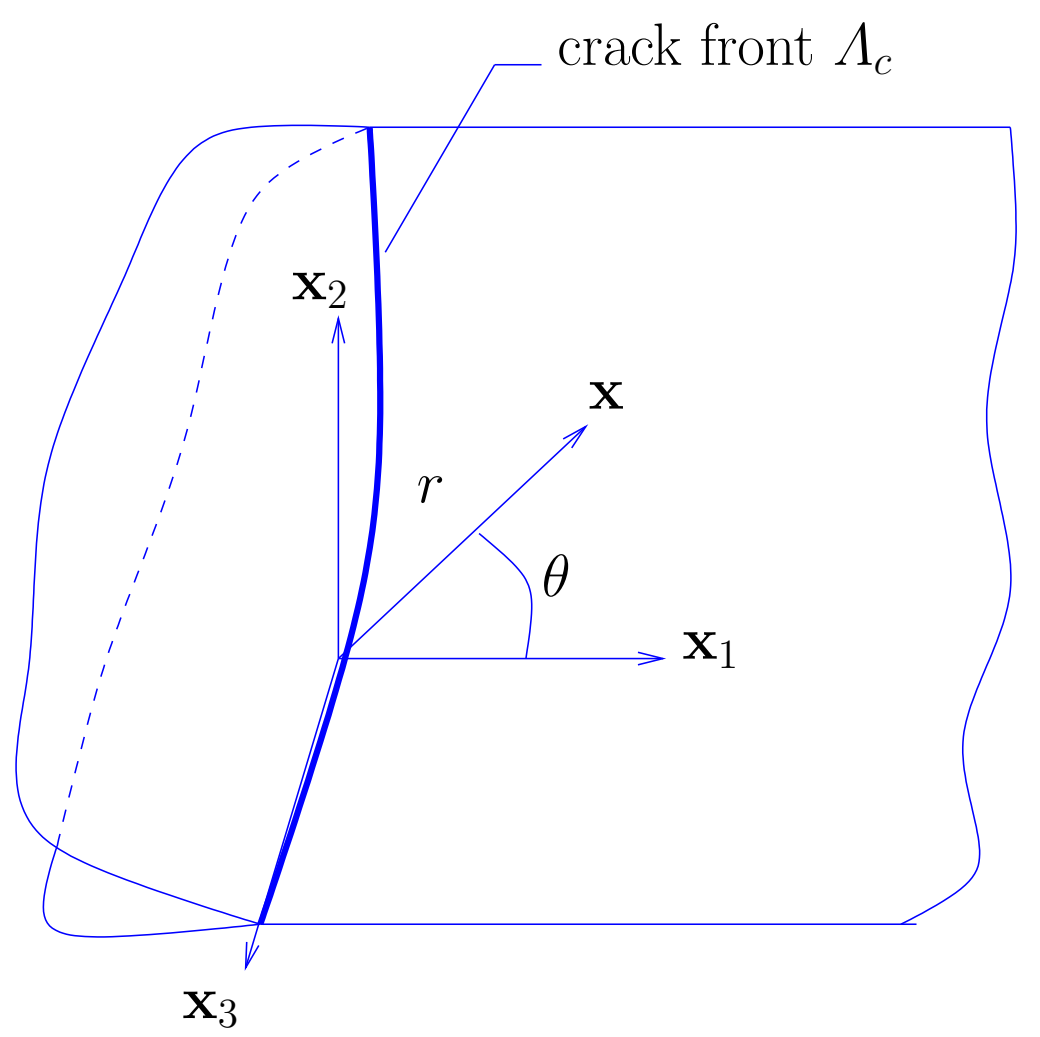

(b) Definition of the crack front coordinate systems for the three dimensional case

Fig. 1 Crack with partially and completely cut particle's domain of influence 
with

$f\left(\mathbf{X}_{I}\right)= \begin{cases}\operatorname{sign}\left[\mathbf{n} \cdot\left(\mathbf{X}_{I}-\mathbf{X}\right)\right] \min \left\|\mathbf{X}_{I}-\mathbf{X}\right\|, & \text { for } \mathbf{X}_{I} \in \mathcal{W}_{b} \\ \mathbf{n} \cdot\left(\mathbf{X}_{\mathrm{tip}}-\mathbf{X}_{I}\right), & \text { for } \mathbf{X}_{I} \in \mathcal{W}_{s}\end{cases}$

where $\mathbf{X}_{t i p}$ are the coordinates of a point on the crack front and $\mathbf{n}$ is the crack normal. Only nodes in $\mathcal{W}_{b}(\mathbf{X})$ are enriched with the additional unknowns $\mathbf{a}$. The third term of equation (3) is applied around the crack front $\mathcal{W}_{s}(\mathbf{X})$. Note that we employed a shifting in Equation (4), denoted by the superimposed bar, to make the enriched region narrower. We know from experiments that for cohesive cracks $\mathbf{B}$ has the form

$\mathbf{B}=\left\{r^{m} \sin \frac{\theta}{2}\right\} \quad m \geq 1$

where $r$ is the minimum distance of $\mathbf{X}$ to the crack front and $\theta(\mathbf{X})=$ $\sin ^{-1}(f / r)(\mathbf{X})$ is the angle between the tangent to the crack surface and the segment $\mathbf{X}-\mathbf{X}_{t i p}$, see Figure 1 .

Remark : since the branch function above vanishes on the crack front, where $r=0$, the approximation is such that the jump in displacement $\llbracket \mathbf{u} \rrbracket$ is zero on the front, i.e. the crack is closed.

Remark : since $\sin \frac{\theta}{2}$ is discontinuous when the angle $\theta$ changes from $\pi$ to $-\pi$, the branch function in Equation 7 used for the front nodes is compatible with the discontinuous function $\bar{H}$ used for the interior nodes, thereby ensuring that the discontinuity is preserved at the front-enriched nodes.

The crack can be represented by different techniques, e.g level sets in Moes et al. [37]; Ventura et al. [51]; Belytschko et al. [9]. However, we do not see any advantage of using level sets and represent the crack explicitly with plane crack surfaces that cross an entire background cell. The signed distance $f$ is computed with respect to these crack surfaces.

When two cracks are joining, the crack tip enrichment is removed for the fronts which disappear in the merging. By using the signed distance functions of the pre-existing and approaching crack, the signed distance function of the approaching crack is modified, see figure 2. Three different subdomains have to be considered: $\left(f_{1}<0, f_{2}<0\right),\left(f_{1}>0, f_{2}>0\right),\left(f_{1}>0, f_{2}<0\right)$ as in figure $2 \mathrm{~b}$ or $\left(f_{1}>0, f_{2}<0\right),\left(f_{1}>0, f_{2}>0\right),\left(f_{1}<0, f_{2}<0\right)$ as in figure $2 \mathrm{~d}$. The signed distance function of crack 1 of a point $\mathbf{X}$ is then obtained by

$f_{1}(\mathbf{X})=\left\{\begin{array}{l}f_{1}^{0}(\mathbf{X}), \text { if } f_{2}^{0}\left(\mathbf{X}_{1}\right) f_{2}^{0}(\mathbf{X})>0 \\ f_{2}^{0}(\mathbf{X}), \text { if } f_{2}^{0}\left(\mathbf{X}_{\mathbf{1}}\right) f_{2}^{0}(\mathbf{X})<0\end{array}\right.$

in which the superscript ${ }^{0}$ stands for the function before junction, [55]. The final approximation without the cross term reads:

$$
\begin{aligned}
\mathbf{u}(\mathbf{X}) & =\sum_{I \in \mathcal{W}(\mathbf{X})} \Phi_{I}(\mathbf{X}) \mathbf{u}_{I}+\sum_{n=1}^{n_{c}} \sum_{I \in \mathcal{W}_{b}(\mathbf{X})} \Phi_{I}(\mathbf{X}) \bar{H}\left(f_{I}^{(n)}(\mathbf{X})\right) \mathbf{a}_{I}^{(n)} \\
& +\sum_{m=1}^{m_{t}} \sum_{I \in \mathcal{W}_{s}(\mathbf{X})} \Phi_{I}(\mathbf{X}) \sum_{K} \overline{\mathbf{B}}_{K}^{(m)}(\mathbf{X}) \mathbf{b}_{K I}^{(m)}
\end{aligned}
$$




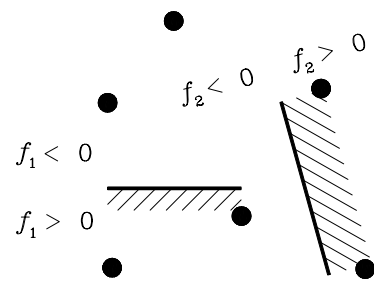

(a)

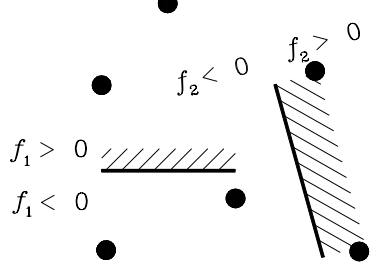

(c)

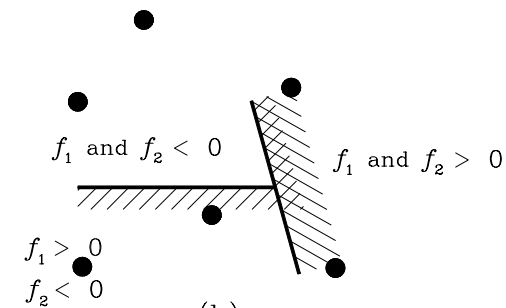

(b)

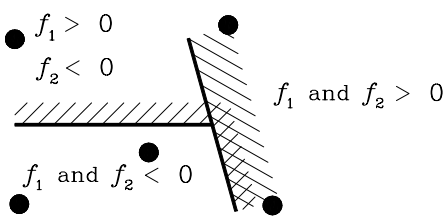

(d)

Fig. 2 Sign functions for crack junction

where $n_{c}$ and $m_{t}$ are, respectively, the number of cracks that completely or partially cross the domain of influence of the corresponding particle. The test functions have the same structure.

We use the element-free Galerkin method with linear complete shape functions of the form:

$\Phi_{I}(\mathbf{X})=\mathbf{p}(\mathbf{X})^{T} \mathbf{A}(\mathbf{X})^{-1} \mathbf{D}\left(\mathbf{X}_{I}\right)$

$\mathbf{A}(\mathbf{X})=\sum_{I} \mathbf{p}\left(\mathbf{X}_{I}\right) \mathbf{p}^{T}\left(\mathbf{X}_{I}\right) W\left(\mathbf{X}-\mathbf{X}_{I}, h\right)$

$\mathbf{D}\left(\mathbf{X}_{I}\right)=\mathbf{p}\left(\mathbf{X}_{I}\right) W\left(\mathbf{X}-\mathbf{X}_{I}, h\right)$

where $\mathbf{p}=(1, X, Y, Z)$ are linear complete base polynomials, $W$ is the kernel function, and $h$ is the size of the domain of influence, also called dilation parameter.

\section{GOVERNING EQUATIONS}

The problem statement is given in Figure 3 .

The strong form of the momentum equation in the updated Lagrangian description is given by

$\varrho \ddot{\mathbf{u}}=\nabla \cdot \boldsymbol{\sigma}+\varrho \mathbf{b}$ in $\Omega \backslash \Gamma^{c}$

with boundary conditions:

$\mathbf{u}(\mathbf{X}, t)=\overline{\mathbf{u}}(\mathbf{X}, t) \quad$ on $\quad \Gamma^{u}$ 


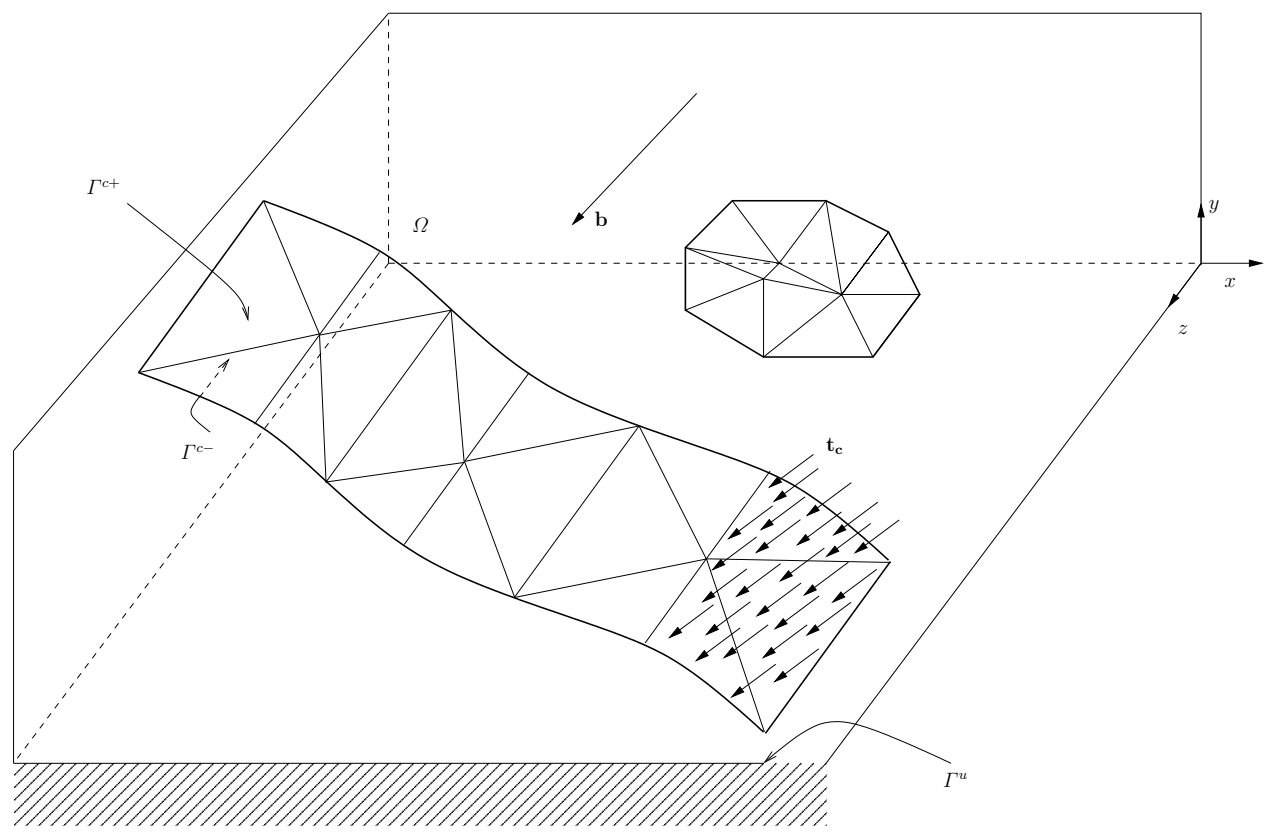

Fig. 3 Problem statement for multiple crack initiation and propagation in threedimensional space. The + and - faces of the cracks are represented. Note that the cracks are represented by the union of contiguous triangular and quadrangular planar segments: the section of the tetrahedral background cells by the cracking plane.

$\mathbf{n} \cdot \boldsymbol{\sigma}(\mathbf{X}, t)=\overline{\mathbf{t}}(\mathbf{X}, t) \quad$ on $\quad \Gamma^{t}$

$\mathbf{n} \cdot \boldsymbol{\sigma}^{-}=-\mathbf{n} \cdot \boldsymbol{\sigma}^{+}=\mathbf{t}_{c}$ on $\Gamma^{c}$

where $\varrho$ is the current mass density, $\ddot{\mathbf{u}}$ is the acceleration, $\boldsymbol{\sigma}$ are the Cauchy stresses $^{3}, \mathbf{b}$ designates the body force, $\overline{\mathbf{u}}$ and $\overline{\mathbf{t}}$ are the prescribed displacement and traction, respectively, $\mathbf{n}$ is the outward normal to the domain and $\Gamma^{u} \bigcup \Gamma^{t} \bigcup \Gamma^{c}=\Gamma,\left(\Gamma^{u} \bigcap \Gamma^{t}\right) \bigcup\left(\Gamma^{t} \bigcap \Gamma^{c}\right) \bigcup\left(\Gamma^{c} \bigcap \Gamma^{u}\right)=\varnothing$. Moreover, we assume that the stresses $\boldsymbol{\sigma}$ at the crack surface $\Gamma^{c}$ are bounded. Since the stresses are not well defined on the crack, the crack surface $\Gamma^{c}$ is excluded from domain $\Omega$ which is considered an open set. Refer to Figure 3 for a graphical representation.

\section{THE DISCRETE MOMENTUM EQUATION}

In the equations below, the superscripts $u, a$, and $b$ denote the standard, discontinuous and near-tip parts respectively. In the matrix operators, when

\footnotetext{
3 the superscripts + and - designate the face of the crack under consideration, as shown in Figure 3
} 
mixed terms appear, they are noted, for instance $u a$, meaning "mixed standarddiscontinuous term". The subscripts int and ext stand for internal and external forces, respectively. Upper-case indices such as $I, J$ denote degree of freedom numbers.

The weak form of the momentum equation is given by

$\delta W=\delta W_{i n t}-\delta W_{e x t}+\delta W_{k i n}=0$

where

$\delta W_{i n t}=\int_{\Omega \backslash \Gamma^{c}}(\nabla \otimes \delta \mathbf{u}): \boldsymbol{\sigma} d \Omega$

$\delta W_{e x t}=\int_{\Omega \backslash \Gamma^{c}} \varrho \delta \mathbf{u} \cdot \mathbf{b} d \Omega+\int_{\Gamma^{t}} \delta \mathbf{u} \cdot \overline{\mathbf{t}} d \Gamma+\int_{\Gamma^{c}} \llbracket \delta \mathbf{u} \rrbracket \cdot \mathbf{t}_{c} d \Gamma$

$\delta W_{k i n}=\int_{\Omega \backslash \Gamma^{c}} \varrho \delta \mathbf{u} \cdot \ddot{\mathbf{u}} d \Omega$

Substituting the test and trial functions into eqs. (18) to (20), we obtain

$$
\begin{aligned}
& \delta W_{k i n}=\sum_{I} \delta \mathbf{u}_{I} \sum_{J} \int_{\Omega \backslash \Gamma^{c}} \varrho \Phi_{I}(\mathbf{X}) \cdot \Phi_{J}(\mathbf{X}) d \Omega \ddot{\mathbf{u}}_{J} \\
&+\sum_{I} \delta \mathbf{u}_{I} \sum_{J} \sum_{n} \int_{\Omega \backslash \Gamma^{c}} \varrho \Phi_{I}(\mathbf{X}) \cdot \Phi_{J}(\mathbf{X}) \bar{H}\left(f_{J}^{(n)}(\mathbf{X})\right) d \Omega \ddot{\mathbf{a}}_{J}^{(n)} \\
&+\sum_{I} \delta \mathbf{u}_{I} \sum_{J} \sum_{m} \int_{\Omega \backslash \Gamma^{c}} \varrho \Phi_{I}(\mathbf{X}) \cdot \Phi_{J}(\mathbf{X}) \overline{\mathbf{B}}_{K}^{(m)} d \Omega \ddot{\mathbf{b}}_{K J}^{(m)} \\
&+\sum_{I} \sum_{n} \delta \mathbf{a}_{I}^{(n)} \sum_{J} \int_{\Omega \backslash \Gamma^{c}} \varrho\left[\Phi_{I}(\mathbf{X}) \bar{H}\left(f_{I}^{(n)}(\mathbf{X})\right)\right] \cdot \Phi_{J}(\mathbf{X}) d \Omega \ddot{\mathbf{u}}_{J} \\
&+\sum_{I} \sum_{n} \delta \mathbf{a}_{I}^{(n)} \sum_{J} \sum_{N} \int_{\Omega \backslash \Gamma^{c}} \varrho\left[\Phi_{I}(\mathbf{X}) \bar{H}\left(f_{I}^{(n)}(\mathbf{X})\right)\right] \cdot\left[\Phi_{J}(\mathbf{X}) \bar{H}\left(f_{J}^{(N)}(\mathbf{X})\right)\right] d \Omega \ddot{\mathbf{a}}_{J}^{(N)} \\
&+\sum_{I} \sum_{n} \delta \mathbf{a}_{I}^{(n)} \sum_{J} \sum_{m} \int_{\Omega \backslash \Gamma^{c}} \varrho\left[\Phi_{I}(\mathbf{X}) \bar{H}\left(f_{I}^{(n)}(\mathbf{X})\right)\right] \cdot\left[\Phi_{J}(\mathbf{X}) \overline{\mathbf{B}}_{K}^{(m)}\right] d \Omega \ddot{\mathbf{b}}_{K J}^{(m)} \\
&+\sum_{I} \sum_{m} \delta \mathbf{b}_{R I}^{(m)} \sum_{J} \int_{\Omega \backslash \Gamma^{c}} \varrho\left[\Phi_{I}(\mathbf{X}) \bar{B}_{R}^{(m)}\right] \cdot \Phi_{J}(\mathbf{X}) d \Omega \ddot{\mathbf{u}}_{J} \\
&+\sum_{I} \sum_{m} \delta \mathbf{b}_{R I}^{(m)} \sum_{J} \sum_{n} \int_{\Omega \backslash \Gamma^{c}} \varrho\left[\Phi_{I}(\mathbf{X}) \bar{B}_{R}^{(m)}\right] \cdot\left[\Phi_{J}(\mathbf{X}) \bar{H}\left(f_{J}^{(n)}(\mathbf{X})\right)\right] d \Omega \ddot{\mathbf{a}}_{J}^{(n)} \\
&+\sum_{I} \sum_{m} \delta \mathbf{b}_{R I}^{(m)} \sum_{J} \sum_{M} \int_{\Omega \backslash \Gamma^{c}} \varrho\left[\Phi_{I}(\mathbf{X}) \bar{B}_{R}^{(m)}\right] \cdot\left[\Phi_{J}(\mathbf{X}) \bar{B}_{K}^{(M)}\right] d \Omega \ddot{\mathbf{b}}_{K J}^{(M)} \\
&+\sum_{I} \delta \mathbf{u}_{I} \int_{\Omega \backslash \Gamma^{c}} \nabla \Phi_{I}(\mathbf{X}) \cdot \boldsymbol{\sigma} d \Omega \\
&+\sum_{I} \sum_{n} \delta \mathbf{a}_{I}^{(n)} \int_{\Omega \backslash \Gamma^{c}}\left[\nabla \Phi_{I}(\mathbf{X}) \bar{H}\left(f_{I}^{(n)}(\mathbf{X})\right)+\nabla \bar{H}\left(f_{I}^{(n)}(\mathbf{X})\right) \Phi_{I}(\mathbf{X})\right] \cdot \boldsymbol{\sigma} d \Omega \\
& \delta W_{i n t}
\end{aligned}
$$




$$
\begin{aligned}
& +\sum_{I} \sum_{m} \delta \mathbf{b}_{K I}^{(m)} \int_{\Omega \backslash \Gamma^{c}}\left[\nabla \Phi_{I}(\mathbf{X}) \bar{B}_{K}^{(m)}+\nabla \bar{B}_{K}^{(m)} \Phi_{I}(\mathbf{X})\right] \cdot \boldsymbol{\sigma} d \Omega \\
& \delta W_{e x t}=\sum_{I} \delta \mathbf{u}_{I} \int_{\Omega \backslash \Gamma^{c}} \varrho \Phi_{I}(\mathbf{X}) \cdot \mathbf{b} d \Omega \\
& +\sum_{I} \delta \mathbf{u}_{I} \int_{\Gamma^{t}} \Phi_{I}(\mathbf{X}) \cdot \overline{\mathbf{t}} d \Gamma \\
& +\sum_{I} \sum_{n} \delta \mathbf{a}_{I}^{(n)} \int_{\Omega \backslash \Gamma^{c}} \varrho\left[\Phi_{I}(\mathbf{X}) \bar{H}\left(f_{I}^{(n)}(\mathbf{X})\right)\right] \cdot \mathbf{b} d \Omega \\
& +\sum_{I} \sum_{n} \delta \mathbf{a}_{I}^{(n)} \int_{\Gamma^{t}}\left[\Phi_{I}(\mathbf{X}) \bar{H}\left(f_{I}^{(n)}(\mathbf{X})\right)\right] \cdot \overline{\mathbf{t}} d \Gamma \\
& +\sum_{I} \sum_{m} \delta \mathbf{b}_{K I}^{(m)} \int_{\Omega \backslash \Gamma^{c}} \varrho\left[\Phi_{I}(\mathbf{X}) \bar{B}_{K}^{(m)}\right] \cdot \mathbf{b} d \Omega \\
& +\sum_{I} \sum_{m} \delta \mathbf{b}_{K I}^{(m)} \int_{\Gamma^{t}}\left[\Phi_{I}(\mathbf{X}) \bar{B}_{K}^{(m)}\right] \cdot \overline{\mathbf{t}} d \Gamma \\
& +\sum_{I} \sum_{n} \int_{\Gamma^{c}} \llbracket \delta \mathbf{a}_{I}^{(n)}\left[\Phi_{I}(\mathbf{X}) \bar{H}\left(f_{I}^{(n)}(\mathbf{X})\right)\right] \rrbracket \cdot \mathbf{t}_{c} d \Gamma \\
& +\sum_{I} \sum_{m} \int_{\Gamma^{c}} \llbracket \delta \mathbf{b}_{K I}^{(m)}\left[\Phi_{I}(\mathbf{X}) \bar{B}_{K}^{(m)}\right] \rrbracket \cdot \mathbf{t}_{c} d \Gamma
\end{aligned}
$$

After some algebraic operations the final form of the equation of motion is

$\mathbf{M}_{I J} \cdot \ddot{\mathbf{u}}_{I}=\mathbf{F}_{I}^{\text {ext }}-\mathbf{F}_{I}^{\text {int }}$

with

$$
\begin{aligned}
& \mathbf{M}_{I J}=\left[\begin{array}{lll}
\mathbf{m}_{I J}^{u u} & \mathbf{m}_{I J}^{u a} & \mathbf{m}_{I J}^{u b} \\
\mathbf{m}_{I J}^{a u} & \mathbf{m}_{I J}^{a a} & \mathbf{m}_{I J}^{a b} \\
\mathbf{m}_{I J}^{b u} & \mathbf{m}_{I J}^{b a} & \mathbf{m}_{I J}^{b b}
\end{array}\right] \\
& \ddot{\mathbf{u}}_{I}=\left[\begin{array}{c}
\ddot{\mathbf{u}}_{I}^{u} \\
\ddot{\mathbf{a}}_{I} \\
\ddot{\mathbf{b}}_{I K}
\end{array}\right] \\
& \mathbf{F}_{I}^{e x t}=\left[\begin{array}{c}
\mathbf{f}_{I}^{u, e x t} \\
\mathbf{f}_{I}^{a, e x t} \\
\mathbf{f}_{I K}^{b, e x t}
\end{array}\right] \\
& \mathbf{F}_{I}^{i n t}=\left[\begin{array}{l}
\mathbf{f}_{I}^{u, i n t} \\
\mathbf{f}_{I}^{a, i n t} \\
\mathbf{f}_{I K}^{b, i n t}
\end{array}\right]
\end{aligned}
$$

with 


$$
\begin{aligned}
& \mathbf{m}_{I J}^{u u}=\int_{\Omega \backslash \Gamma^{c}} \varrho \Phi_{I}(\mathbf{X}) \Phi_{J}(\mathbf{X}) d \Omega \\
& \mathbf{m}_{I J}^{u a}=\int_{\Omega \backslash \Gamma^{c}} \varrho \Phi_{I}(\mathbf{X}) \Phi_{J}(\mathbf{X}) \bar{H}\left(f_{I}^{(n)}(\mathbf{X})\right) d \Omega=\mathbf{m}_{I J}^{a u} \\
& \mathbf{m}_{I J}^{u b}=\int_{\Omega \backslash \Gamma^{c}} \varrho \Phi_{I}(\mathbf{X}) \Phi_{J}(\mathbf{X}) \overline{\mathbf{B}}_{K}^{(m)} d \Omega=\mathbf{m}_{I J}^{b u} \\
& \mathbf{m}_{I J}^{a a}=\int_{\Omega \backslash \Gamma^{c}} \varrho \Phi_{I}(\mathbf{X}) \bar{H}\left(f_{I}^{(n)}(\mathbf{X})\right) \Phi_{J}(\mathbf{X}) \bar{H}\left(f_{I}^{(n)}(\mathbf{X})\right) d \Omega \\
& \mathbf{m}_{I J}^{a b}=\int_{\Omega \backslash \Gamma^{c}} \varrho \Phi_{I}(\mathbf{X}) \bar{H}\left(f_{I}^{(n)}(\mathbf{X})\right) \Phi_{J}(\mathbf{X}) \overline{\mathbf{B}}_{K}^{(m)} d \Omega=\mathbf{m}_{I J}^{b a} \\
& \mathbf{m}_{I J}^{b b}=\int_{\Omega \backslash \Gamma^{c}} \varrho \Phi_{I}(\mathbf{X}) \overline{\mathbf{B}}_{K}^{(m)} \Phi_{J}(\mathbf{X}) \overline{\mathbf{B}}_{K}^{(m)} d \Omega \\
& \mathbf{f}_{I}^{u, e x t}=\int_{\Omega \backslash \Gamma^{c}} \varrho \mathbf{b} \Phi_{I}(\mathbf{X}) d \Omega+\int_{\Gamma^{t}} \overline{\mathbf{t}} \Phi_{I}(\mathbf{X}) d \Gamma+\mathbf{f}_{I}^{u, c r} \\
& \mathbf{f}_{I}^{a, e x t}=\int_{\Omega \backslash \Gamma^{c}} \varrho \mathbf{b} \Phi_{I}(\mathbf{X}) \bar{H}\left(f_{I}^{(n)}(\mathbf{X})\right) d \Omega+\int_{\Gamma^{t}} \overline{\mathbf{t}} \Phi_{I}(\mathbf{X}) \bar{H}\left(f_{I}^{(n)}(\mathbf{X})\right) d \Gamma+\mathbf{f}_{I}^{a, c r} \\
& \mathbf{f}_{I}^{b, e x t}=\int_{\Omega \backslash \Gamma^{c}} \varrho \mathbf{b} \Phi_{I}(\mathbf{X}) \overline{\mathbf{B}}_{K}^{(m)} d \Omega+\int_{\Gamma^{t}} \overline{\mathbf{t}} \Phi_{I}(\mathbf{X}) \overline{\mathbf{B}}_{K}^{(m)} d \Gamma+\mathbf{f}_{I}^{b, c r} \\
& \mathbf{f}_{I}^{u, i n t}=\int_{\Omega \backslash \Gamma^{c}} \nabla \Phi_{I}(\mathbf{X}) \cdot \boldsymbol{\sigma} d \Omega \\
& \mathbf{f}_{I}^{a, i n t}=\int_{\Omega \backslash \Gamma^{c}}\left(\left(\nabla \Phi_{I}(\mathbf{X}) \bar{H}\left(f_{I}^{(n)}(\mathbf{X})\right)+\Phi_{I}(\mathbf{X}) \nabla \bar{H}\left(f_{I}^{(n)}(\mathbf{X})\right)\right) \cdot \boldsymbol{\sigma}\right) d \Omega \\
& \mathbf{f}_{I K}^{b, i n t}=\int_{\Omega \backslash \Gamma^{c}}\left(\left(\nabla \Phi_{I}(\mathbf{X}) \overline{\mathbf{B}}_{K}^{(m)}+\Phi_{I}(\mathbf{X}) \nabla \overline{\mathbf{B}}_{K}^{(m)}\right) \cdot \boldsymbol{\sigma}\right) d \Omega \\
& \mathbf{f}_{I}^{a, c r}=\int_{\Gamma_{0}^{c}} \Phi_{I}(\mathbf{X}) \llbracket \bar{H}\left(f_{I}^{(n)}(\mathbf{X})\right) \rrbracket \mathbf{t}_{c} d \Gamma \\
& \mathbf{f}_{I}^{b, c r}=\int_{\Gamma_{0}^{c}} \Phi_{I}(\mathbf{X}) \llbracket \overline{\mathbf{B}}_{K}^{(m)} \rrbracket \mathbf{t}_{c} d \Gamma
\end{aligned}
$$

Equation (25) is the consistent mass matrix. In Equation (31), the spatial derivatives of $\bar{H}$ vanish since the domain is considered as open set. The cohesive forces are taken into account in the external forces, Equation (30). Gauß quadrature is used to obtain the discrete equations where the nodes are usually located on the vertices of the background cells (Figure 4).

The usual subtriangulation procedures are employed to accurately integrate cracked background cells; see Figure 5 e.g. Bordas and Legay [12]; Bordas and Moran [11]. The crack is initiated and propagated through an entire background cell, so that the crack front is composed of line segments lying on the faces of background cells. 


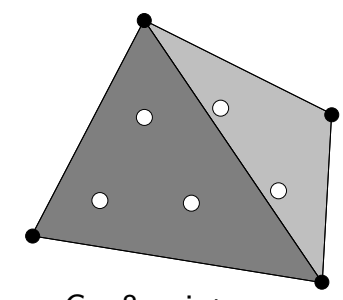

Gauß point

- Field node/particle

Fig. 4 Tetrahedral background cell, field nodes/particles, and Gauß points.

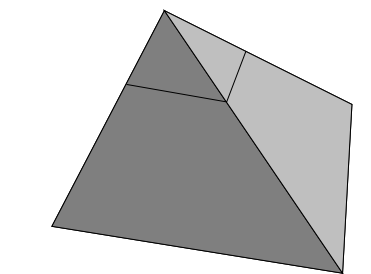

Subdivision of the o
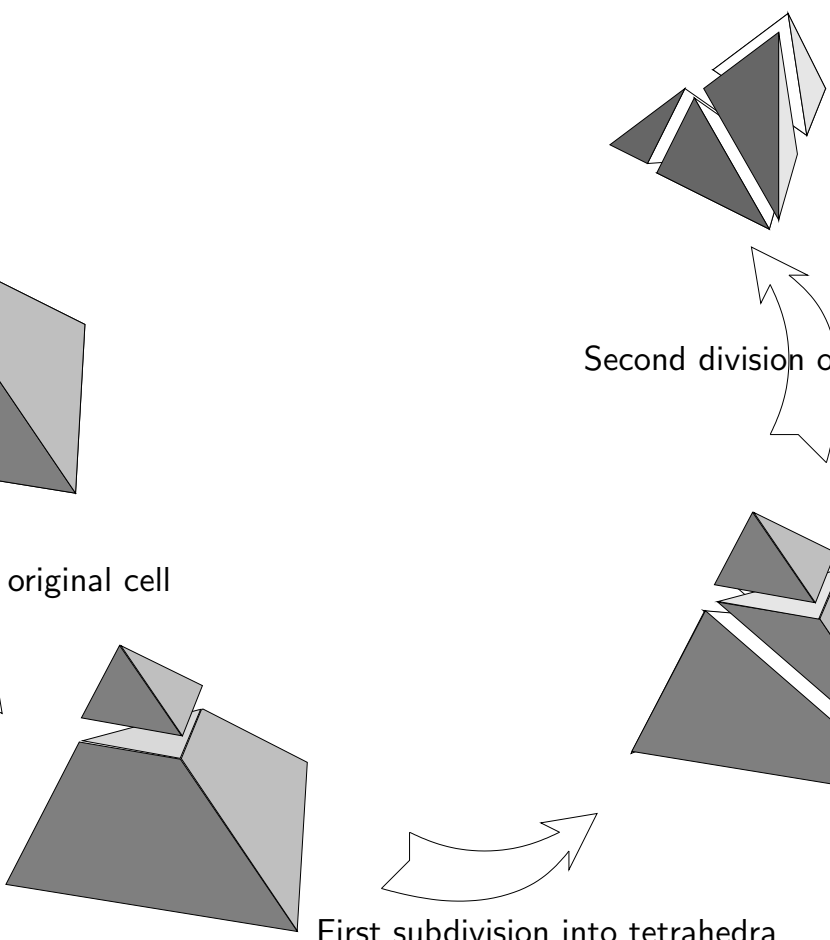

Second division of each tetrahedron:
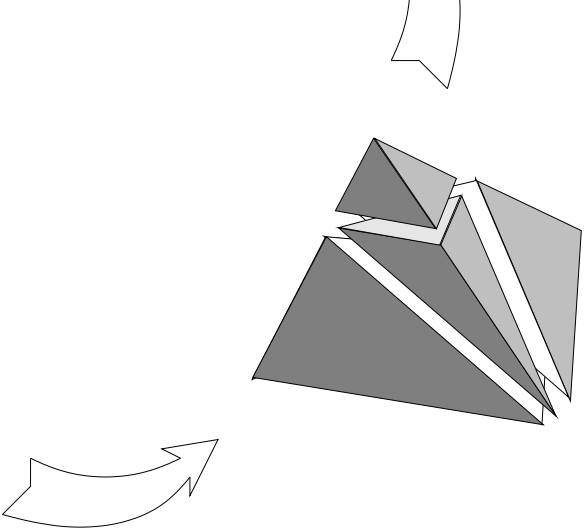

First subdivision into tetrahedra

Fig. 5 Decomposition of tetrahedra split by a discontinuity for accurate integration of the weak form.

\section{$5 h$-adaptive refinement}

We use $h$-adaptivity to obtain a better resolution around the crack front. A certain refinement around the crack tip is essential for the quality of the results. Due to the lack of a mesh, meshfree methods are ideally suited for adaptive procedures. Our adaptivity scheme is based on the estimation of the approximation error. Other error estimators can be found e.g. in Monk [40]; Beck et al. [5]; Houston et al. [24]. Areas with high strain gradients are 

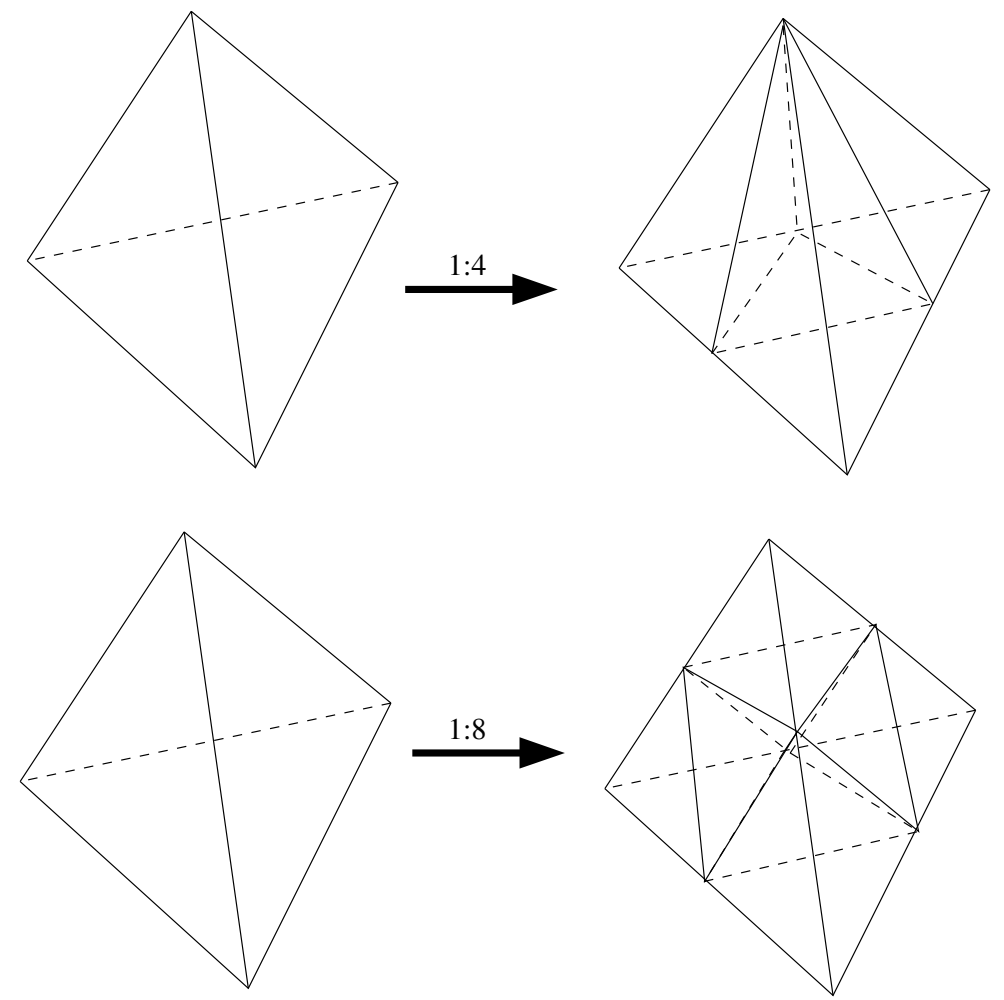

Fig. $6 h$-adaptive refinement strategy according to Loehner [35]

refined, see Rabczuk and Belytschko [44]. The new particles are added so that they span a new tetrahedron, see Figure 6. New Gauß cells are created such that the particles form these Gauß cells, Figure 6. All data is mapped onto the new particle arrangement by Moving Least Squares (MLS) fit. The refinement strategy for an unstructured particle arrangement is shown in figure 6 according to Loehner [35]. We usually used the $1: 8$ refinement because it yields tetrahedra with better aspect ratios. Note that we follow the Devloo-Oden rule [18], so that too coarse a mesh never meets too fine a mesh.

\section{CONSTITUTIVE MODELS}

\subsection{Continuum Models}

The stress in the domain except the crack surface is obtained from continuum constitutive models. We use three constitutive models: Lemaitre's damage model, a neo-Hookean model and a Johnson-Cook plasticity model. 
For the Lemaitre [29] model, the stress-strain behavior is

$\boldsymbol{\sigma}=(1-D) \mathbf{C}_{0}: \boldsymbol{\epsilon}$

where $D$ is a scalar damage variable which ranges from 0 to a maximum of 1 and $\mathbf{C}_{0}$ is the initial elasticity tensor. The damage evolution depends on the effective strain $\bar{\epsilon}$ :

$D(\bar{\epsilon})=1-(1-A) \epsilon_{D_{0}} \bar{\epsilon}^{-1}-A e^{-B\left(\bar{\epsilon}-\epsilon_{D_{0}}\right)}$

with

$\bar{\epsilon}=\sqrt{\sum_{i=1}^{3} \epsilon_{i}^{2} \mathcal{H}\left(\epsilon_{i}\right)}$

where $\epsilon_{i}$ are the principal strains and

$\mathcal{H}(x)=1$ if $x>0$

$\mathcal{H}(x)=0$ if $x<0$

$A, B$ and $\epsilon_{D_{0}}$ are material parameters.

The neo-Hookian model is based on the following potential:

$\Psi(\mathbf{C})=\kappa(\ln J)^{2}+0.5 \nu\left(\bar{I}_{1}-3\right)$

with material parameters $\kappa$ and $\nu, \mathbf{C}=\mathbf{F}^{T} \mathbf{F}$ is the right Cauchy-Green strain tensor and $\bar{I}_{1}=\operatorname{tr} \overline{\mathbf{C}}$ with $\overline{\mathbf{C}}=J^{-2 / 3} \mathbf{C}$. For further details, see Gasser and Holzapfel [21]

We also use the Johnson Cook [25] constitutive model with J2 plasticity. The effective yield stress of the Johnson-Cook model is given by

$\sigma_{Y}=\left(A+B \gamma^{n}\right)\left(1+C \ln \dot{\epsilon}^{*}\right)\left(1-T^{*}\right)$

with material parameters $A, B, C, \dot{\epsilon}^{*}=\dot{\epsilon} / \dot{\epsilon}_{0}$ where $\epsilon$ is the effective plastic strain and $\dot{\epsilon}_{0}$ is the reference strain rate taken to be $1.0 / \mathrm{s}$ and

$T^{*}=\frac{T-T_{r}}{T_{m}-T_{r}}$

where $T_{r}$ is the reference temperature and $T_{m}$ is the melting temperature. We assume that the plastic deformation is completely transformed into heat, so $\beta=1$ for the temperature update:

$\Delta T=\int_{0}^{\gamma} \frac{\beta}{\varrho c_{v}} \sigma_{Y} d \gamma$

where $c_{v}$ is the specific heat and $\varrho$ the current mass density.

6.2 Cohesive laws

The jump in the displacement is governed only by the enrichment and is given by

$\llbracket \mathbf{u}(\mathbf{X}) \rrbracket=2 \sum_{n=1}^{n_{c}} \sum_{I \in W_{b}(\mathbf{X})} \Phi_{I}(\mathbf{X}) \mathbf{q}_{I}+\sum_{m=1}^{m_{t}} \sum_{I \in W_{s}(\mathbf{X})} \Phi_{I}(\mathbf{X}) \sum_{K} \llbracket \overline{\mathbf{B}}_{K}(\mathbf{X}) \rrbracket \mathbf{b}_{K}(41)$ 
The normal part $\delta_{n}$, i.e. the crack opening and the tangential part $\llbracket \mathbf{u}(\mathbf{X}) \rrbracket_{\tau}$, the crack sliding, is given by

$\delta_{n}=\llbracket \mathbf{u}(\mathbf{X}) \rrbracket_{n}=\mathbf{n} \cdot \llbracket \mathbf{u}(\mathbf{X}) \rrbracket$

$\delta_{t}=\llbracket \mathbf{u}(\mathbf{X}) \rrbracket_{\tau}=\|\llbracket \mathbf{u}(\mathbf{X}) \rrbracket-(\llbracket \mathbf{u}(\mathbf{X}) \rrbracket \cdot \mathbf{n}) \mathbf{n}\|$

We adopt the exponential cohesive law by Gasser and Holzapfel [21], that is based on the following potential:

$\Psi\left(i_{1}, i_{4}, \delta\right)=\frac{t_{0}}{2 \delta} \exp \left(-a \delta^{b}\right)\left(i_{4}+\alpha\left(i_{1}-i_{4}\right)\right)$

with $i_{1}=\mathbf{I}:(\tilde{\mathbf{u}} \otimes \tilde{\mathbf{u}})$ and $i_{4}=(\tilde{\mathbf{u}} \otimes \tilde{\mathbf{u}}):(\mathbf{n} \otimes \mathbf{n})$ with material parameters $t_{0}, a, b$ and $\alpha$. With $\mathbf{t}=\frac{\partial \Psi}{\partial \tilde{\mathbf{u}}}$, the cohesive law reads

$\mathbf{t}=\frac{t_{0}}{\delta} \exp \left(-a \delta^{b}\right)\left(\tilde{\mathbf{u}}_{n}+\alpha \tilde{\mathbf{u}}_{t}\right)$

with $\tilde{\mathbf{u}}_{n}=\mathbf{n} \delta_{n}$ and $\tilde{\mathbf{u}}_{t}=\delta_{t} \frac{\tilde{\mathbf{u}}-\tilde{\mathbf{u}}_{n}}{\left|\tilde{\mathbf{u}}-\tilde{\mathbf{u}}_{n}\right|}$. With the damage surface $\Phi(\tilde{\mathbf{u}}, \delta)=$ ||$\tilde{\mathbf{u}}|-\delta|$, the condition that the value of the internal variable is monotonically increasing, $\delta=\max [|\tilde{\mathbf{u}}|]_{t_{0}}^{t}$ and the evolution of the internal variable $\delta$ :

$\dot{\delta}=|\dot{\tilde{\mathbf{u}}}|$

the cohesive law is completely established. No interpenetration condition of the crack surfaces is enforced by a penalty method.

Note that if $\delta=0, \mathbf{t}$ tends to zero. Therefore, we do not allow $\mathbf{t}$ to exceed a certain threshold that is related to the uniaxial tensile strength.

\section{Cracking algorithm}

\subsection{Crack initiation}

For a rate-independent material, loss of hyperbolicity serves us as a criterion for crack initiation. In the case of a rate-dependent material, the transition criterion to discontinuum is governed by the same condition though we do not talk about loss of hyperbolicty any more, since the equations are regularized. Instead, the transition criterion is called loss of material stability. As shown e.g. in Ogden [41], a material point loses stability if the minimum eigenvalue of the acoustic tensor $\mathbf{Q}$ is smaller or equal to zero:

$\min \operatorname{eig}(\mathbf{Q}) \leq 0$ with $\mathbf{Q}=\mathbf{n} \cdot \mathbf{A} \cdot \mathbf{n}$

where $\mathbf{n}$ is the normal to the crack surface when $\min \operatorname{eig}(\mathbf{Q}) \leq 0$ and $\mathbf{A}=$ $\mathbf{C}^{t}+\boldsymbol{\sigma} \otimes \mathbf{I}$ where $\mathbf{C}^{t}$ is the tangent stiffness. For a Rankine material, a crack is initiated when the principal tensile stress exceeds the tensile strength. The crack is then normal to the direction of the principal tensile stress. Note that the Rankine criterion is a special case of the loss of hyperbolicity condition for mode I fracture.

The smoothed, non local crack normal used to determine the crack direction at a given Gauß point is given by the normalized weighted average 


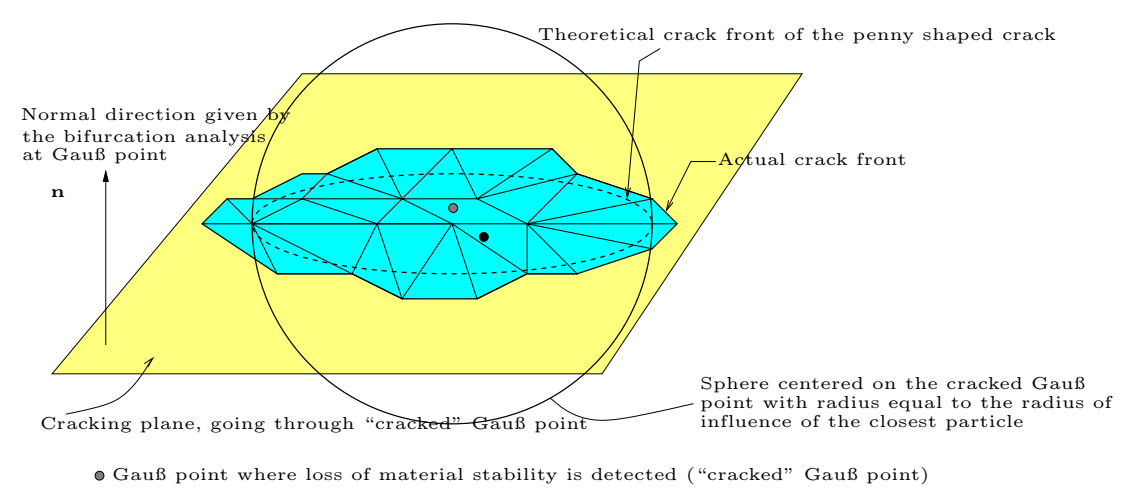

- Closest particle (node) to "cracked" Gauß point

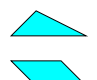

Triangular and quadrangular elementary planar crack surfaces, located in the cracking plane

Fig. 7 Representation of a penny-shaped crack as defined upon crack initiation. Note that the crack is not exactly circular. $h$ is the dilation parameter, i.e. the diameter of the domain of influence of the particle closest to the Gauß point where material instability is detected. Note that the normal, $\mathbf{n}$ is in fact an MLS average of neighboring normals, themselves computed through the discontinuous bifurcation analysis.

of the neighboring normals, where the weight functions are the MLS shape functions:

$\mathbf{n}_{\text {non local }}=\frac{\sum_{J \in \mathcal{S}} \Phi_{J}(\mathbf{X}) \mathbf{n}_{J}}{\left\|\sum_{J \in \mathcal{S}} \Phi_{J}(\mathbf{X}) \mathbf{n}_{J}\right\|}$,

where $\mathcal{S}$ is the set of cells neighboring the cell of interest.

If loss of material stability is detected at a Gauß point, an "almost" this will be explained in the following- penny shaped crack is introduced. We control the crack length, so that the radius of the newly initiated pennyshaped crack is the interpolation radius of the closest particle. If a background cell is crossed by the circle defined by the intersection of the ball of influence of the closest particle and the cracking plane, the cell is assumed cracked. This is illustrated in three dimensions in Figure 7 and, for clarity, in two dimensions, in Figure 8. Possible intersections of a tetrahedron by the crack plane are given in Figure 9a. Due to the fact that the entire background cell is cracked, the crack will not be exactly penny shaped but will depend on the arrangement of the background cells; as is evident in Figure 7. At this point, it becomes obvious that crack initiation depends also on the size of the tetrahedra background cell (and the meshfree dilation parameter). Accuracy is improved significantly by adaptive refinement.

It may happen that several Gauß points lose material stability in a given cell. In this case, the cracking plane is assumed to go through the isobarycenter of the cracked Gauß points, and the normal is taken as the average 


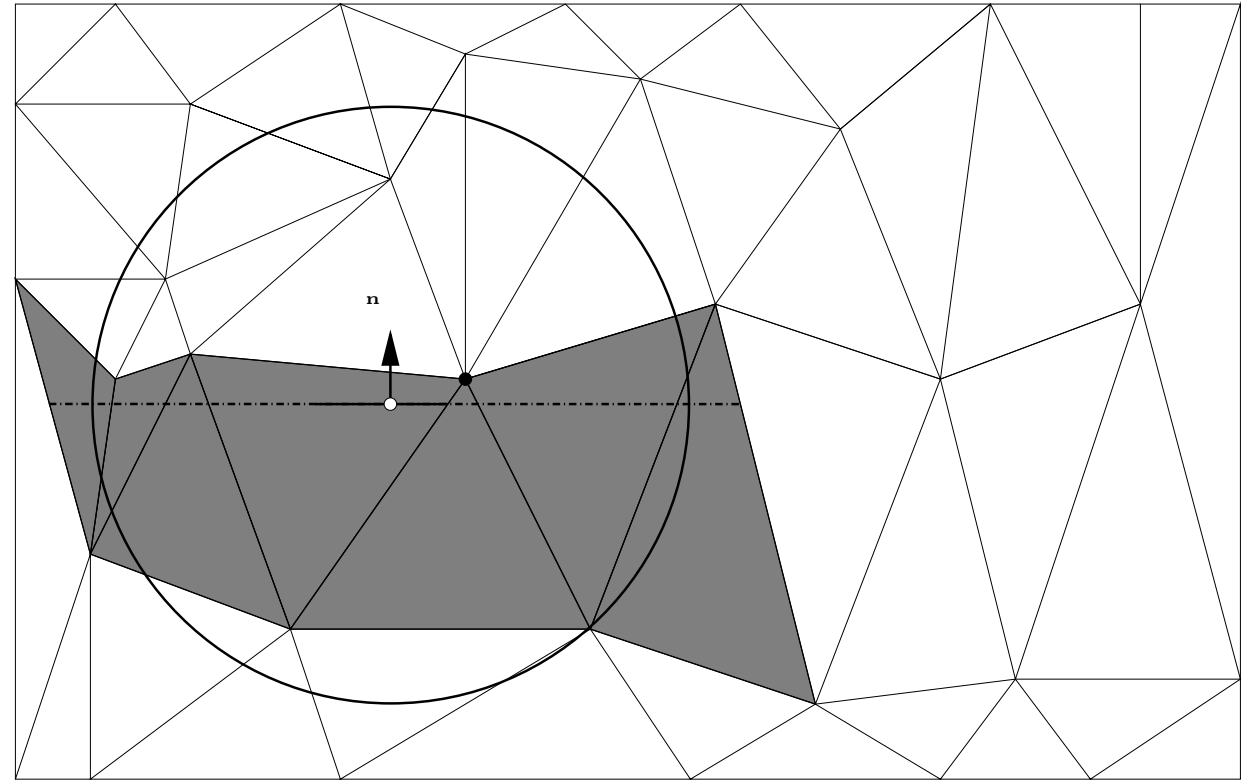

cracked cell

- "cracked" Gauß point, where material instability is detected

- particle (node) closest to "cracked" Gauß point

-....... initiated "penny" crack

Fig. 8 Two-dimensional representation of a penny-shaped crack (here a line segment) as defined upon crack initiation. Note that the crack length is not exactly equal to the radius of influence of the closest node, since all background cells that are intersected by the crack segment are assumed completely cracked. $h$ is the dilation parameter, i.e. the diameter of the domain of influence of the particle closest to the Gauß point where material instability is detected. Note that the normal, $\mathbf{n}$ is in fact a MLS average of neighboring normals, themselves computed through the discontinuous bifurcation analysis.

of the normals associated with each cracked Gauß point. An example of this is shown in Figure 10. If the normals computed at the failing Gauß points are too different, then, this is a sign that a crack is trying to branch in the cell. However, in the examples we treated, this case never happened.

Note that the piecewise linear line used to define the front (tip) enrichment functions is aligned with the edges of the background cells where the crack front closes. A possible front is shown for illustration in Figure 11. The union of the spherical enriched domains centered on points on the crack front forms an enriched tube around the front. We found it advantageous to place enriched nodes at the end of each crack front line. Difficulties arise at sharp corners or kinks at the crack front, since a local coordinate system cannot be defined uniquely at each of the vertices on the front. We use the enrichment to the closest distance to the crack front and did not observe any major difficulties. A smoothing of the crack front shape can alleviate these 


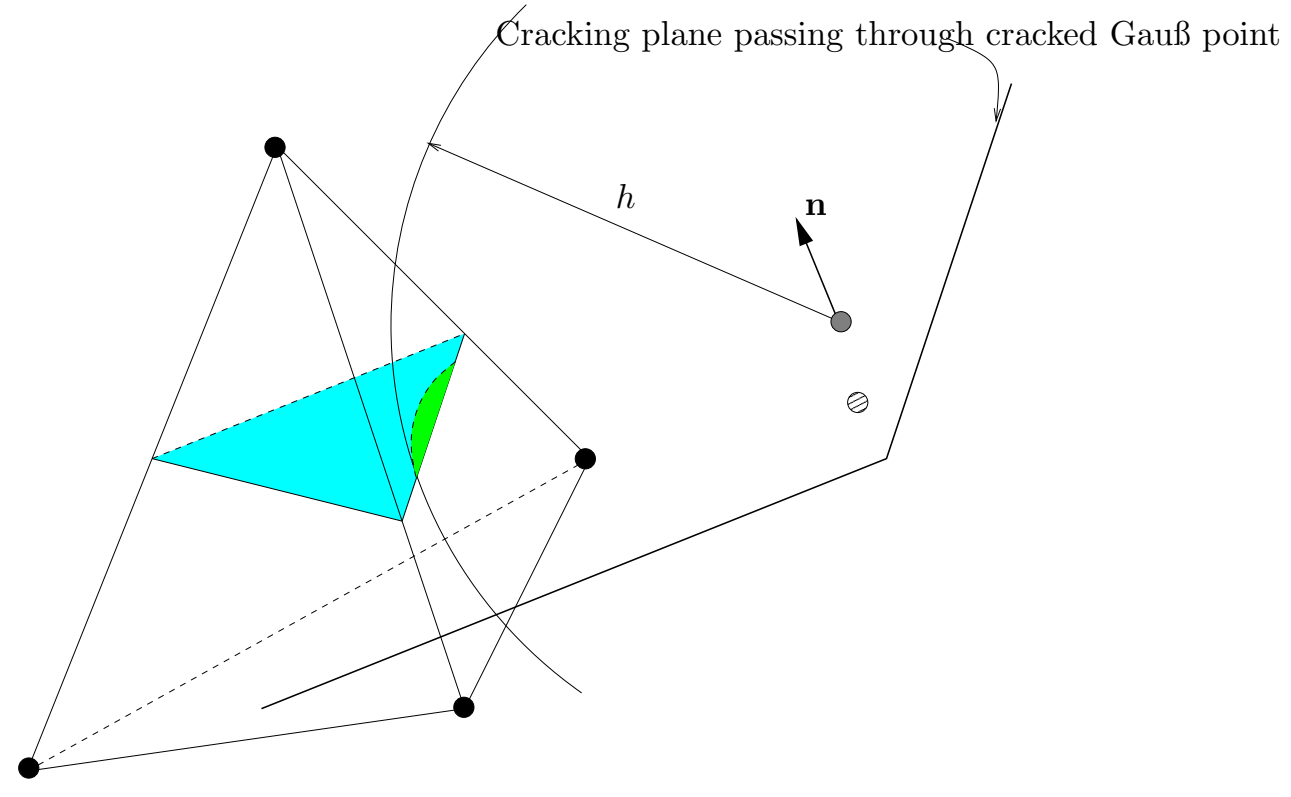

LEGEND

- Cracked Gauß point

- Particle

• Closest particle to cracked Gauß point

Intersection of the domain of influence

with the section of the tetrahedral

cell by the cracking plane

(a) Triangular section

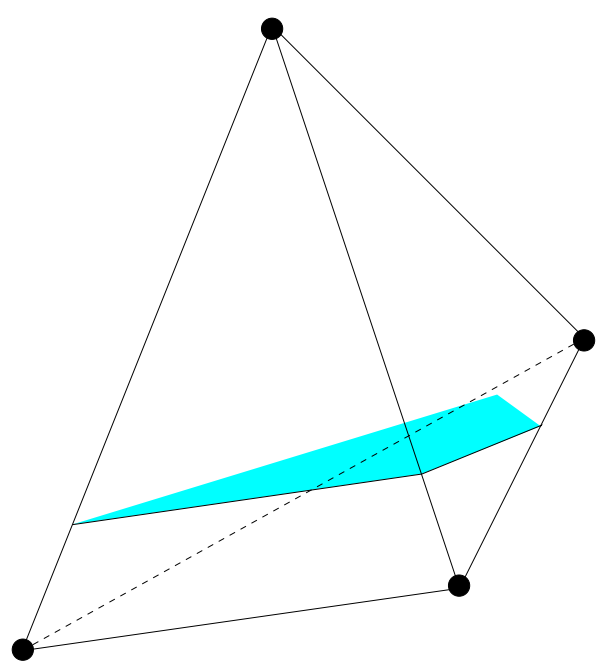

(b) Quadrangular section

Fig. 9 Elementary triangular and quadrangular sections of a tetrahedral cell by the cracking plane, defined by its normal obtained by the bifurcation analysis. 
Plane in which the crack is introduced

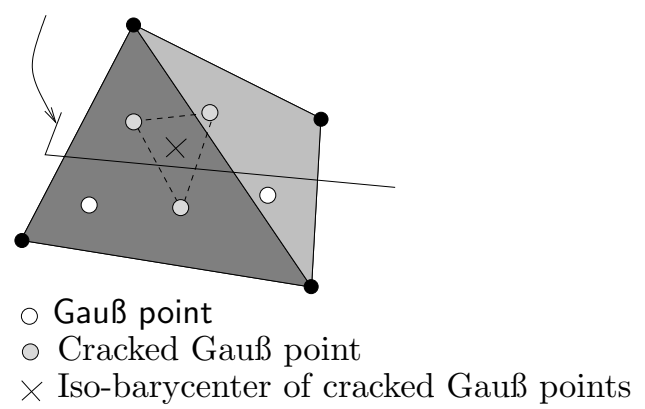

Fig. 10 Crack initiation in the case of several cracked Gauß points in a given cell.

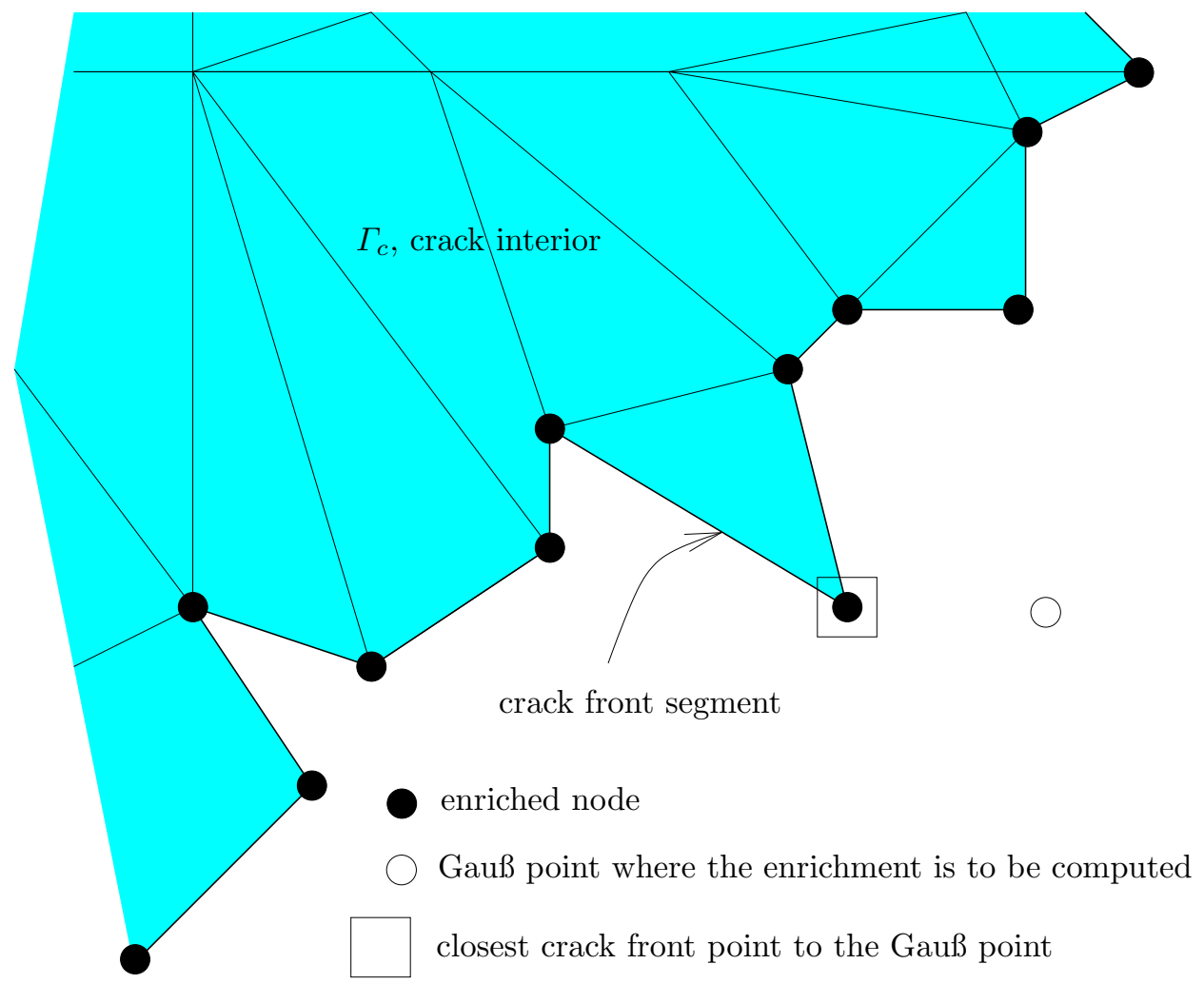

Fig. 11 A possible piecewise linear crack front.

problems. Here, a smooth representation of the crack surface by level sets or non-uniform rational B-splines (NURBS) can definitely be advantageous. Another smooth-level set procedure is described by Duflot [19].

Another possible case is that of cracks initiating and joining simultaneously, as shown in Figures 12 and 13 . 


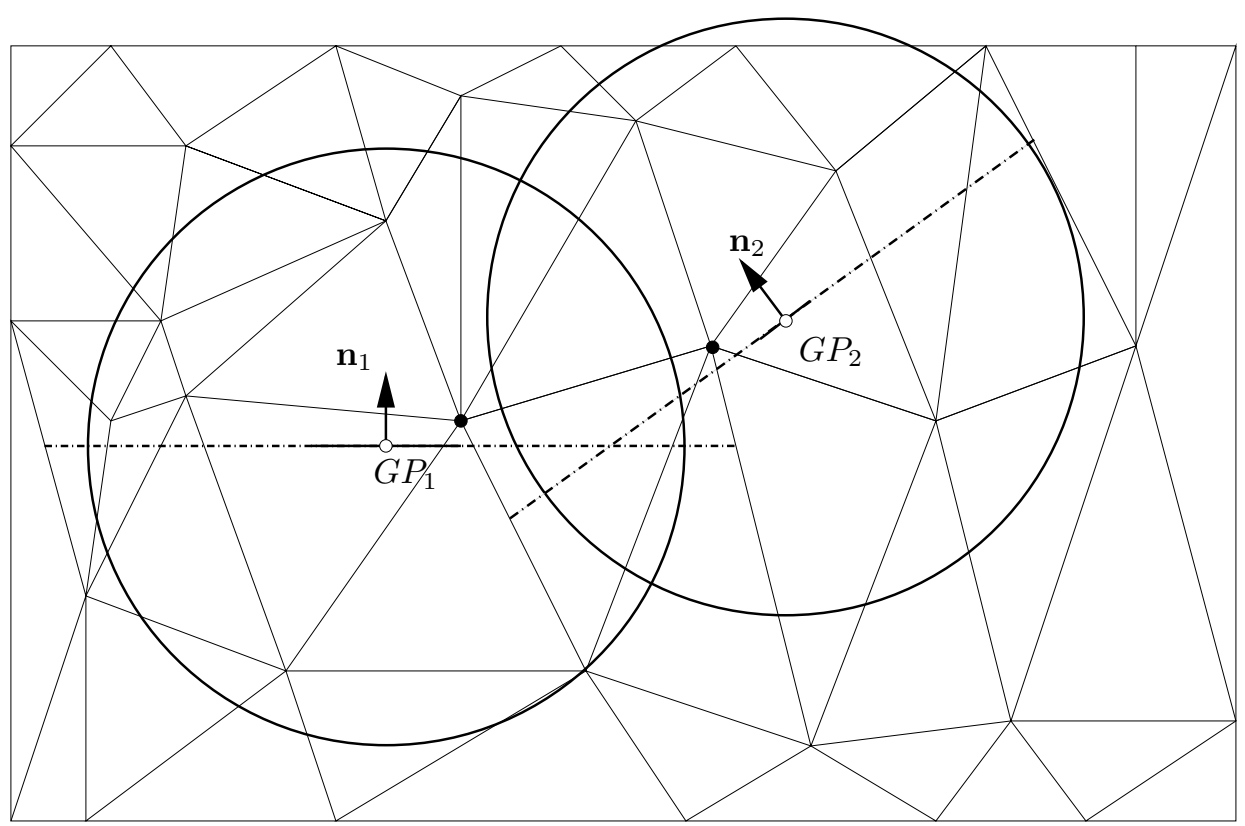

- "cracked" Gauß point, where material instability is detected

- particle (node) closest to "cracked" Gauß point

........ initiated "penny" crack

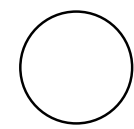

Circle centered on the cracked Gauß point

with radius equal to the size of the domain of

influence of the particle closest to that Gauß point

(a) In simultaneous initiation and joining, this configuration is not allowed.

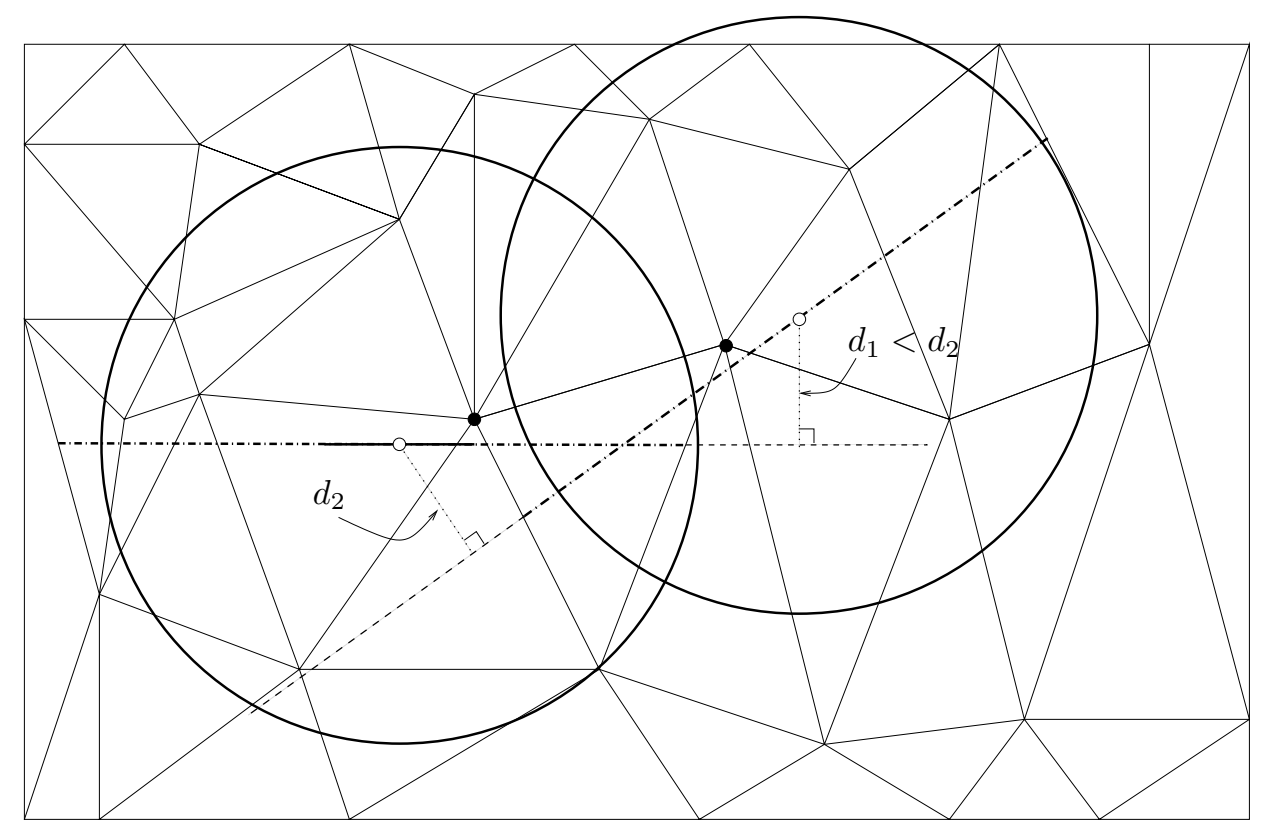

- "cracked" Gauß point, where material instability is detected

- particle (node) closest to "cracked" Gauß point

-......... initiated "penny" crack

(b) Instead, the crack further from the failing Gauß point is cut at the intersection, resulting in the configuration of Figure 13

Fig. 12 Two cracks initiating and joining simultaneously. All cells crossed by the circle, and by the line with normal $\mathbf{n}$ and passing through the failed Gauß point are considered cracked. 


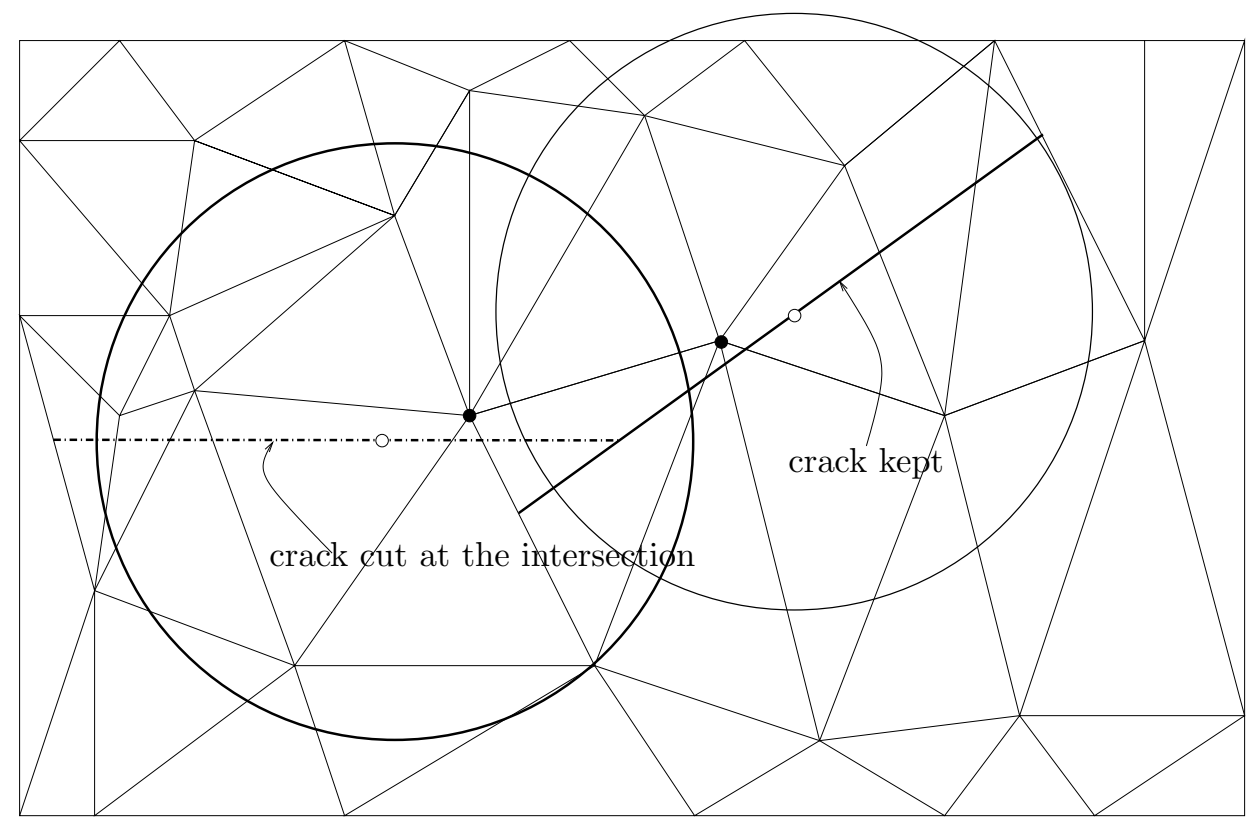

- "cracked" Gauß point, where material instability is detected

- $\quad$ particle (node) closest to "cracked" Gauß point

Fig. 13 Two cracks initiating and joining simultaneously, the one further from the failed Gauß point is cut at the intersection with the other initiating crack.

\subsection{Crack front propagation and crack junctions}

A difficult task in three-dimensions is to trace the crack path, at least if crack path continuity is desired. We propose a simple scheme to propagate and join cracks in three dimensions; crack branching is not explicitly considered as e.g. in Daux et al. [16] and Belytschko et al. [9].

Note, that crack path continuity cannot be ensured when the crack normals obtained by the discontinuous bifurcation analysis differ severely in neighboring background cells. In this case, crack path continuity can generally be enforced only along certain lines and gaps might occur across the faces of the background cells. Such gaps usually lead to a mesh dependency. Other researchers choose to represent the crack path completely continuously as described e.g in Areias and Belytschko [3]. However, if more than one background cell neighboring a given cell of interest is cracked and crack continuity is to be conserved (Figure 7.2), the crack direction in the cell of interest is uniquely determined by the crack normals obtained in its neighboring cells ${ }^{4}$. This direction might not coincide with that obtained through

\footnotetext{
${ }^{4}$ Imagine a tetrahedron with two cracked neighbors. Since crack continuity is to be fully preserved, the elementary planar crack segment in the tetrahedron of interest must be contiguous with the crack segments of its neighbors.
} 


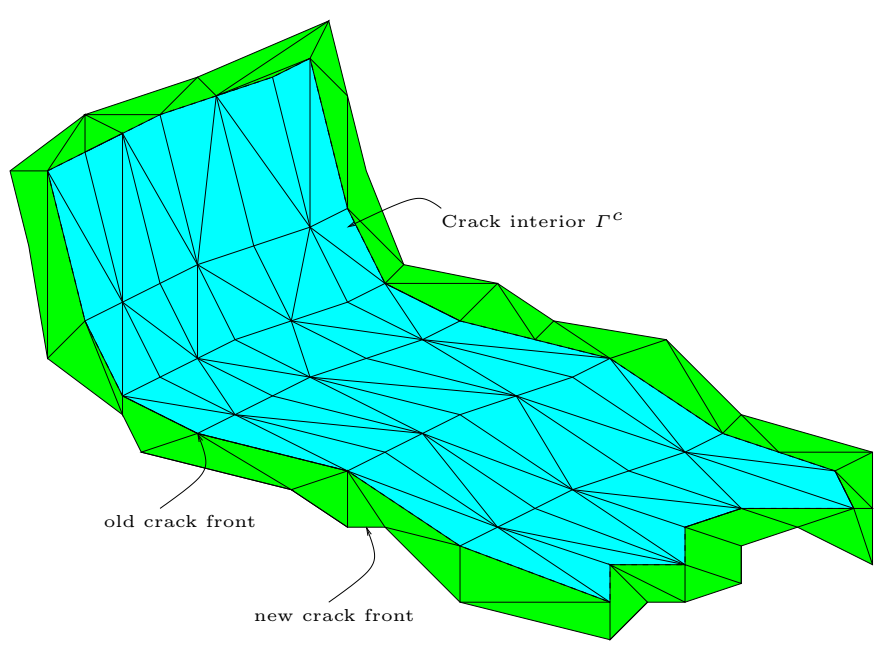

Fig. 14 Propagation of the crack front

the localization/bifurcation analysis in the cell of interest. It becomes apparent that the results might then depend on the order in which background cells crack. Consequently, enforcing "complete" 5 crack path continuity might lead to erroneous results if the crack direction of the localization analysis is ignored. As mentioned above, a smoothing technique is usually sufficient to avoid the need of such procedures. Remember that it is important to enforce crack continuity, since methods that do not enforce crack path continuity completely generally tend to lock and fail to reproduce the correct system response.

There are two possible elemental planar crack surface shapes: triangular and quadrangular, see Figure 9. When two cracks are joining, we assume the propagating crack to stop at the intersection line, as outlined in Section 2 and depicted in Figure 16. In case two propagating or newly created cracks are joining, we always stop the newly created crack and cut the crack at its intersecting line. In rare cases, where two newly initiated cracks join in a tetrahedral cell, as illustrated in Figure 18 simultaneously we compute the distance of the midpoint of the crack plane to the material points where hyperbolicity is lost. The crack surface with smaller distance is kept while the other one will be cut at the intersection, as is shown in Figures 12 and 13. The general algorithm is illustrated in Figure 17. 


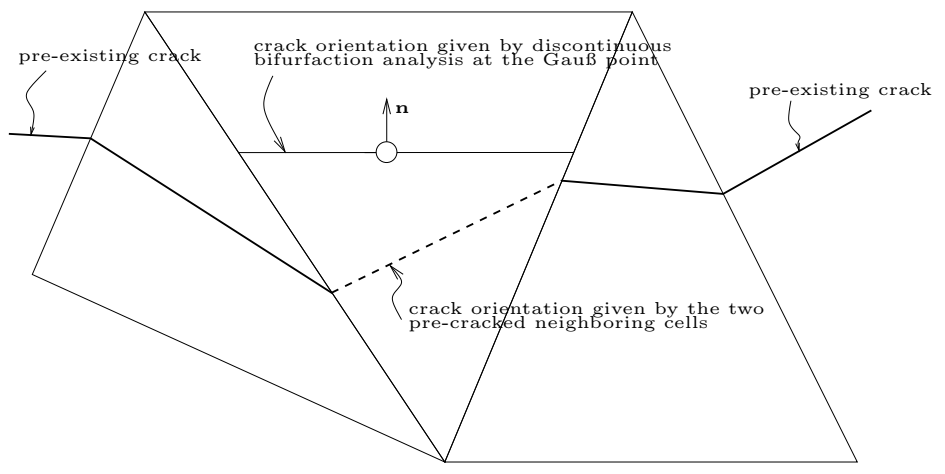

(a)

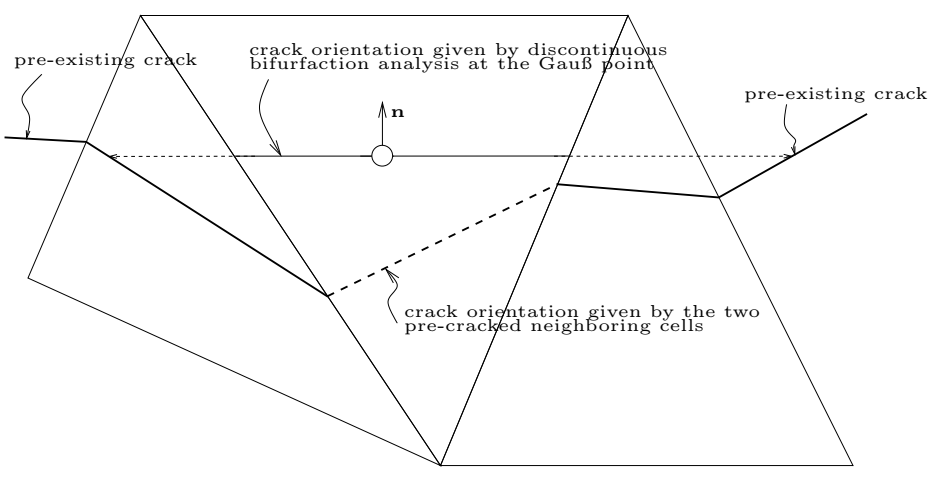

(b)

Fig. 15 Possible incompatibilities between neighboring crack normals, and normals given by the discontinuous bifurcation analysis.

\section{NUMERICAL APPLICATIONS}

8.1 Penny-shaped crack in a finite cube under uniaxial tension

Consider a cube of side length $a$ at the center of which a penny-shaped crack of radius $r$ is embedded. The cube is subjected to uniaxial tension $\sigma_{c}=1 \mathrm{kPa}$. For an infinite plate, the analytical solution for the stress intensity factor is given by $K_{I}=2 \sigma_{c} \sqrt{r / \pi}$. For $a=50 r$, the error in the stress intensity factor is computed for different refinements with different particle spacing $(30 \times 30 \times 30,60 \times 60 \times 60$ and $120 \times 120 \times 120)$; note that the mesh is finer around the crack, see figure 19 in a two dimensional topview. For this example, we use the Westergaard solution for the crack tip enrichment, $\mathbf{B}=$

\footnotetext{
5 in the sense "everywhere"
} 


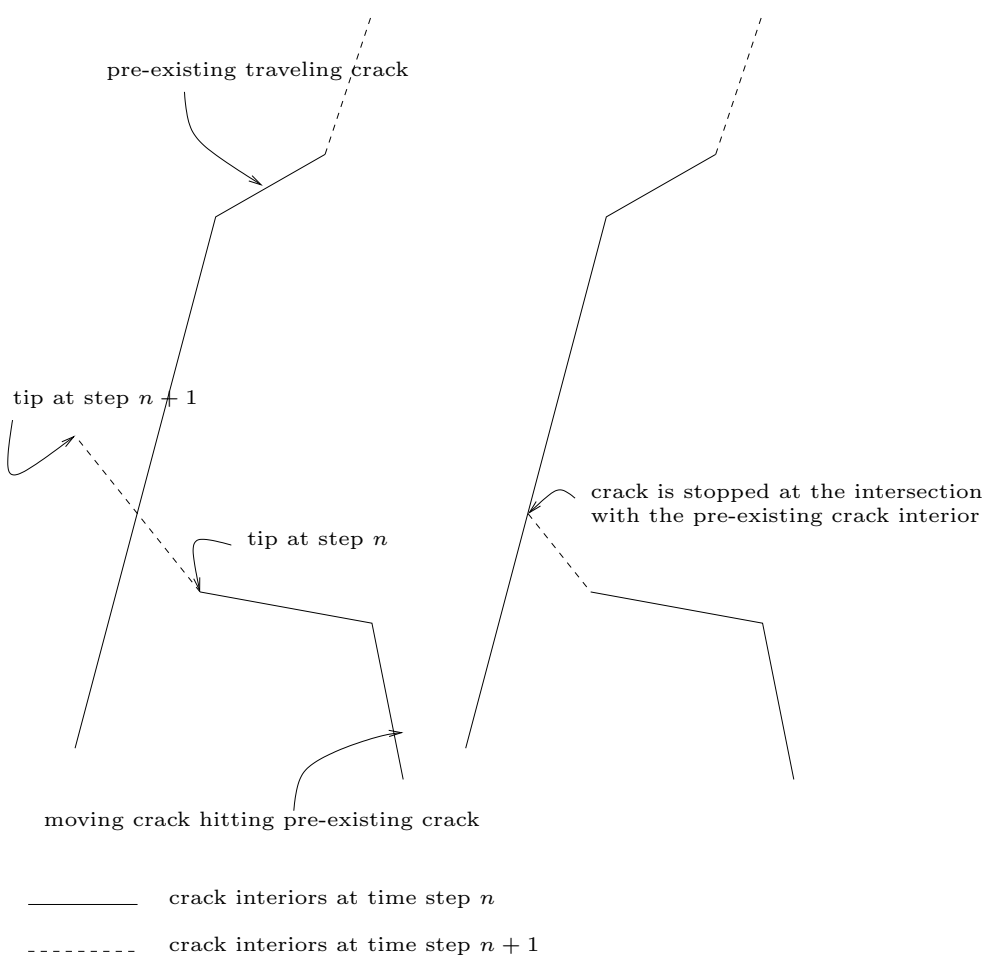

Fig. 16 When two cracks intersect, the moving crack is stopped at the intersection with the existing crack. Configurations such as that of the left of the figure are not allowed.

Table 1 Stress intensity factors for the mode I penny-shaped crack problem

\begin{tabular}{cccc}
\hline Relative error on $K_{I}$ & $30 \times 30 \times 30$ & $60 \times 60 \times 60$ & $120 \times 120 \times 120$ \\
\hline average (\%) & 0.085 & 0.05 & 0.03 \\
maximum (\%) & 0.2 & 0.112 & 0.068 \\
\hline
\end{tabular}

$\left\{\sqrt{r} \sin \frac{\theta}{2}, \sqrt{r} \cos \frac{\theta}{2}, \sqrt{r} \sin \frac{\theta}{2} \sin \theta, \sqrt{r} \cos \frac{\theta}{2} \sin \theta\right\}$. The average error as well as the maximum error is given in Table 1 . The results are excellent.

\subsection{Arrea-Ingraffea beam}

Consider the Arrea and Ingraffea [4] beam that fails in a combined tensileshear mode. The beam is loaded at points $A$ and $B$ according to figure 20. The Young's modulus is 28,000 MPa, tensile strength is $2.8 \mathrm{MPa}$ and Poisson's ration $\nu=0.18$. We have used the Lemaitre [29] damage model in tension and linear elasticity in compression. Loss of hyperbolicity is employed for crack initiation. A linear decaying cohesive law is used with fracture energy $G_{f}=100 \mathrm{~N} / \mathrm{m}$. We tested two different discretizations, starting with approx- 


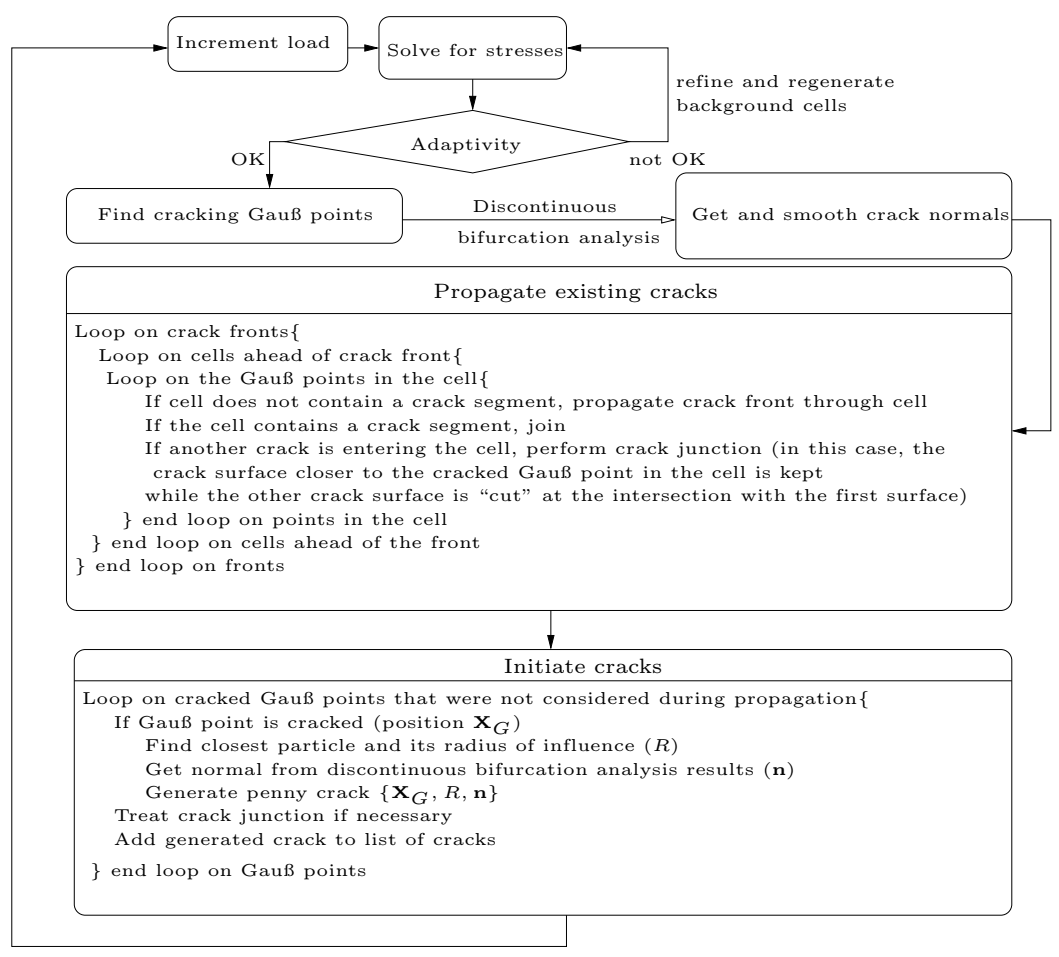

Fig. 17 Algorithm for crack propagation, initiation and junction.

imately 48,000 and 12,000 particles, respectively. Two and three adaptation steps are allowed.

The final crack path for the fine particle distribution is shown in figure $21 \mathrm{a}$, the crack path for the coarse particle discretization is illustrated in figure 21 b. For a better illustration, only a closeup is shown. For two earlier load steps, the crack pattern is shown in figure 22. As can be seen, the crack front is not straight but somewhat irregular, which is also observed experimentally and corroborates the well-known fact that of plane-stress crack fronts are not straight. In the Figure, the crack geometry is represented by a triangulated surface. Of course, the crack surface is also composed of quadrilaterals (Figure 9), but for ease of representation, these quadrilaterals are split into two triangles. The numerical crack pattern is similar to the experimental one and does not show mesh-dependency. The load displacement curve (right of the notch) is shown in Figure 23 and lies in the experimental scatter.

\subsection{Pull-out test}

Consider a pull out test of reinforced concrete as shown in figure 24. This example was studied previously by Gasser and Holzapfel [21] and Areias and Belytschko [3] by the PUFEM and XFEM, respectively. We also employed 


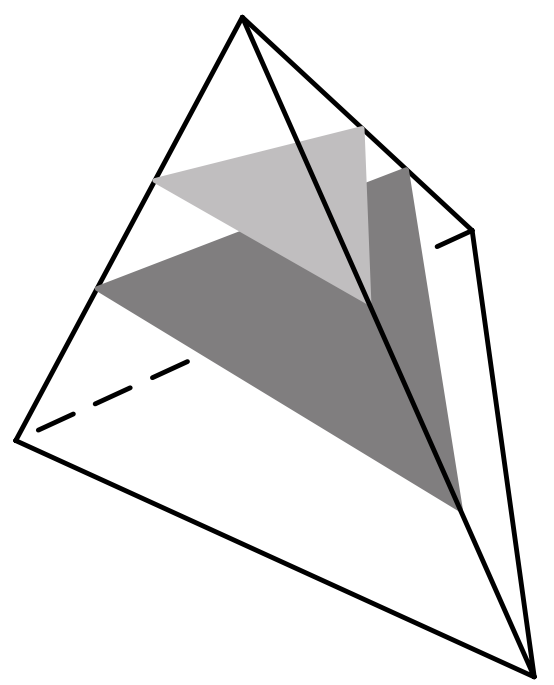

a)

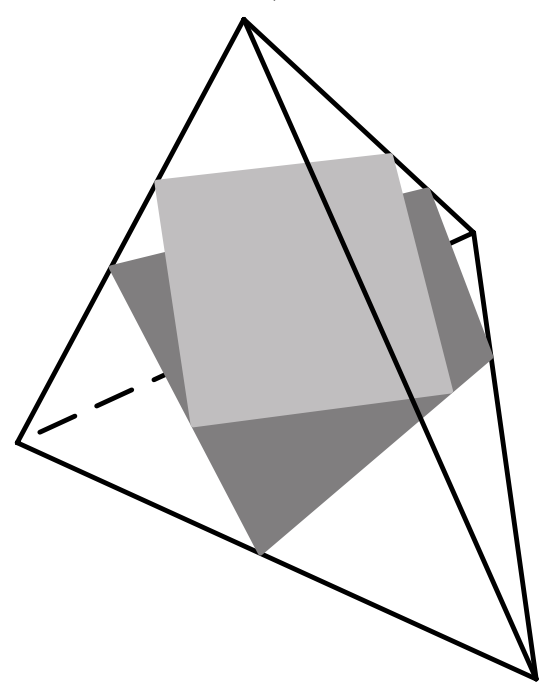

c)

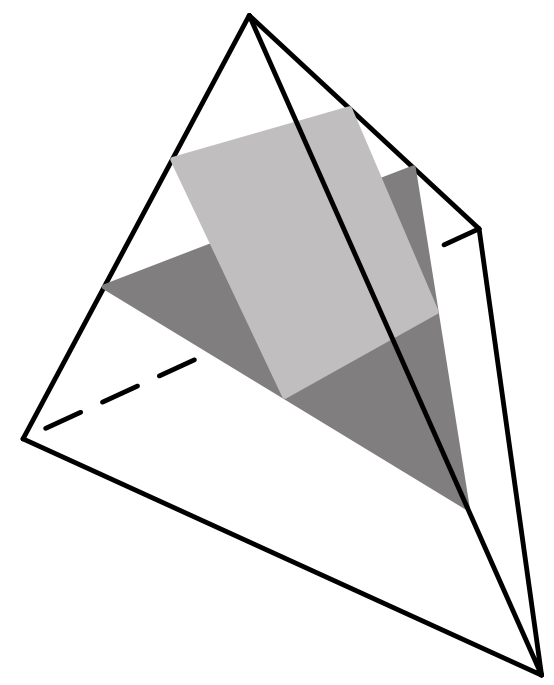

b)

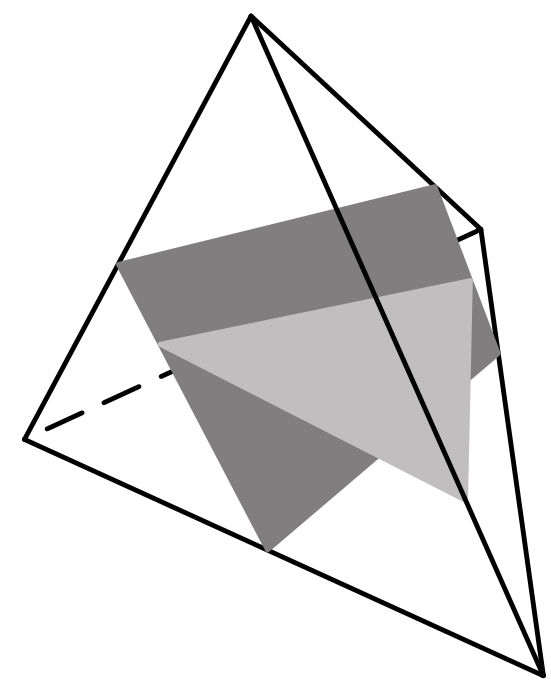

d)

Fig. 18 Crack junction

symmetry conditions and modelled only one quarter of the specimen. A vertical displacement boundary condition is applied to pull out the reinforcement bar out of the concrete specimen as illustrated in figure 24. We run this example on 16 processors. The data was distributed equally on 16 processors (16 equal slices, geometrically) and data transfer is realized using Message Passing Interface (MPI). More details about domain decomposition and solvers can be found e.g. in Alonso and Valli [1, 2]; Hiptmair [23]. Since we expect the crack to propagate over the entire circumference, no redistribution of the 


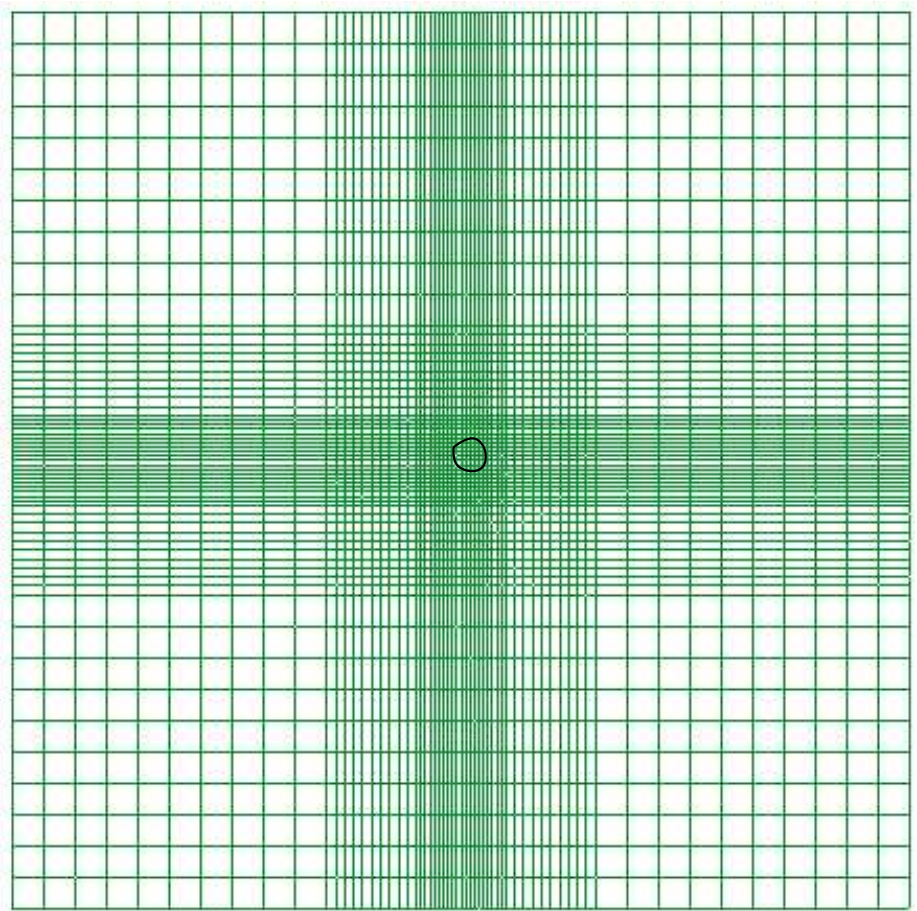

Fig. 19 Typical discretization of the penny crack problem; two dimensional topview

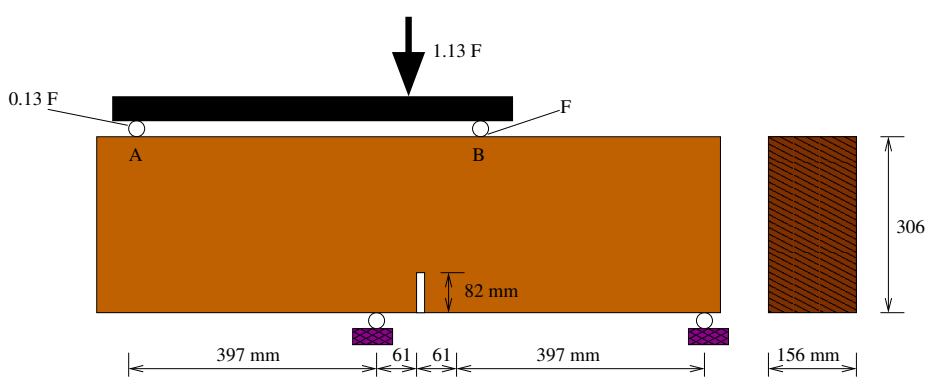

Fig. 20 The tensile/shear beam from Arrea Ingraffea

data is necessary. Redistribution of data in an adaptive parallel computation is one of the trickiest part in the parallel implementation. We adopted the constitutive and cohesive model in Gasser and Holzapfel [21]. The material parameters are $\kappa=16,670 \mathrm{MPa}$ and $\nu=12,500 \mathrm{MPa}$. For the cohesive model, we use, according to [21], $t_{0}=3 \mathrm{MPa}, a=11.323 \mathrm{~mm}^{-1}, b=0.674$ and $\alpha=1$.

We tested two discretization starting with 15,000 and 45,000 nodes. A maximum of three refinement steps are allowed. The crack pattern at different load stages is shown in figure 25 for the finer discretization. The load- 


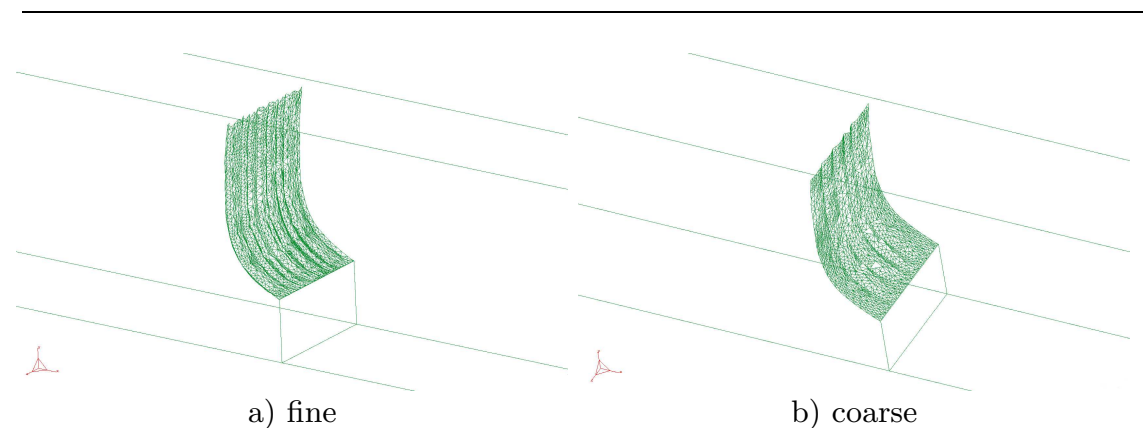

Fig. 21 Closeup of the crack for the Arrea-Ingraffea beam

deflection curve is shown in figure 26 and is similar to the on in Gasser and Holzapfel [21] and Areias and Belytschko [3].

\subsection{Flyer plate impact}

The next example is a flyer plate impact problem. We assume that the flyer plate is of the same material as the target plate. The test-set up is illustrated in figure 27. Experimental data is available from Chevrier and Klepaczko [14]. They varied the material and the impact velocity. We carried out two computations, with an impact velocity of $205 \mathrm{~m} / \mathrm{s}$ and $290 \mathrm{~m} / \mathrm{s}$. In both experiments, spalling was observed through the spall plane for the higher impact velocity was clearly visible and crossed almost the entire specimen.

We use the high strength steel material data according to Chevrier and Klepaczko [14] and varied the strength in the specimen, i.e. we mutliplied the material strength by a factor $0.95<\alpha<1.05$ obtained from a log-normal distribution around the mean value of 1 and a standard deviation of $1.5 \%$. This should account for potential small imperfections.

A quarter of the specimen at the end of the computation is shown in Figure 28 for the two different impact velocities. Note, that we did not use symmetry conditions in the simulation. The spall plane is very similar to that observed in the experiment. It occurs over almost the entire width of the plate and lies almost in the same plane. A topview on the cracks at different time steps is shown in Figure 29a-c for an impact velocity of $290 \mathrm{~m} / \mathrm{s}$. Cracking does not happen "exactly" simultaneously and cracks are propagating and joining. However, the final crack pattern is reached within less than five microseconds. The crack pattern for the lower impact velocity is shown in Figure $29 \mathrm{~d}$. The spall plane is located at the center of the plate, see also Figure 28.

\subsubsection{Kalthoff problem}

Kalthoff and Winkler [26] performed a series of experiments where a steel plate is subjected to impact loading with different impact velocities as shown in Figure 30. For low impact velocities $v_{i}=20 \mathrm{~m} / \mathrm{s}$, a brittle failure occured and two symmetric cracks propagate in a seventy degree angle through the 


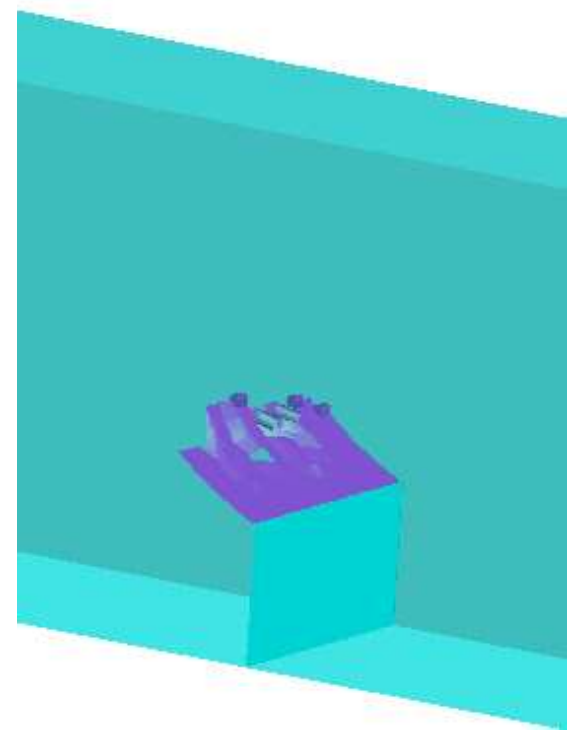

a) coarse

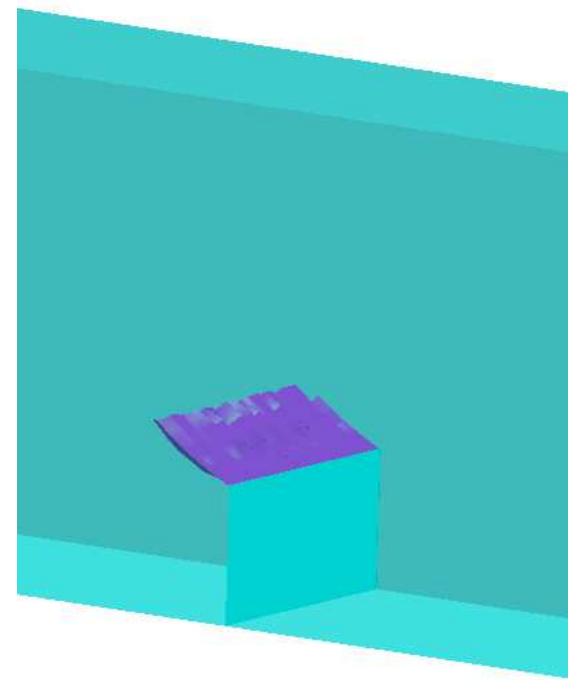

c) fine

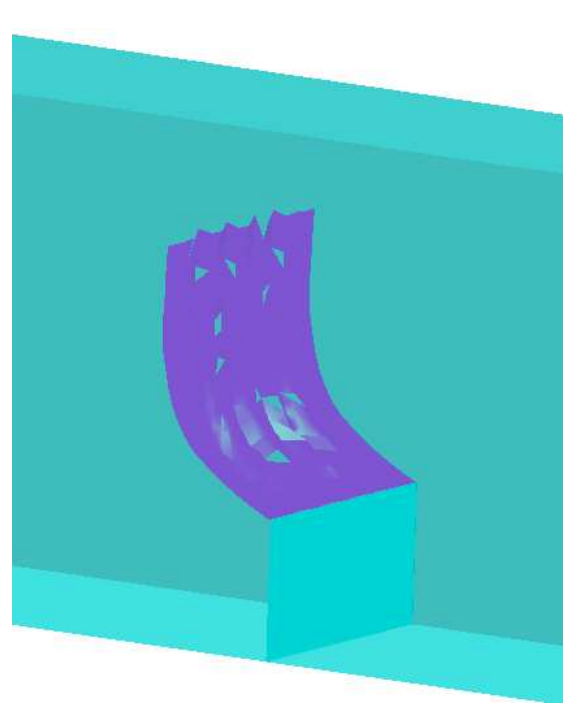

b) coarse

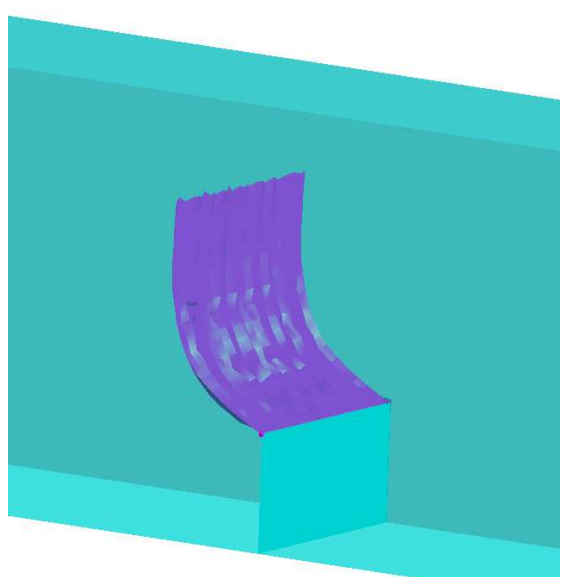

d) fine

Fig. 22 Closeup of the crack for the Arrea-Ingraffea beam at different load steps

entire specimen. We use the Johnson Cook model [25] with material parameters $A=792 \mathrm{MPa}, B=509 \mathrm{MPa}, C=0.014, n=0.26, m=0.55$. The density is $\varrho=7800 \mathrm{~kg} / \mathrm{m}^{3}, K=157 \mathrm{GPa}, \nu=76 \mathrm{GPa}$ and the specific heat is $c_{v}=477 \mathrm{~J} / \mathrm{kg} \mathrm{C}, T_{r}=296 \mathrm{~K}, T_{m}=1033 \mathrm{~K}$ and $\beta=1$. Crack initiation is governed by the Rankine criterion with $f_{t}=2 A$. Figure 31 shows the final crack pattern for two different refinements. In the first computation, we did not use adaptivity while in the second one, we allowed two refinement steps. 


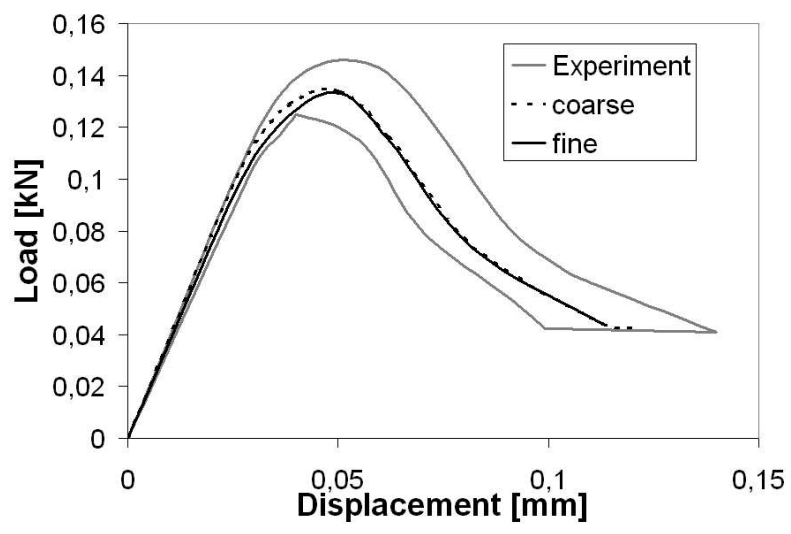

Fig. 23 Load deflection curve of the tensile/shear beam from Arrea Ingraffea

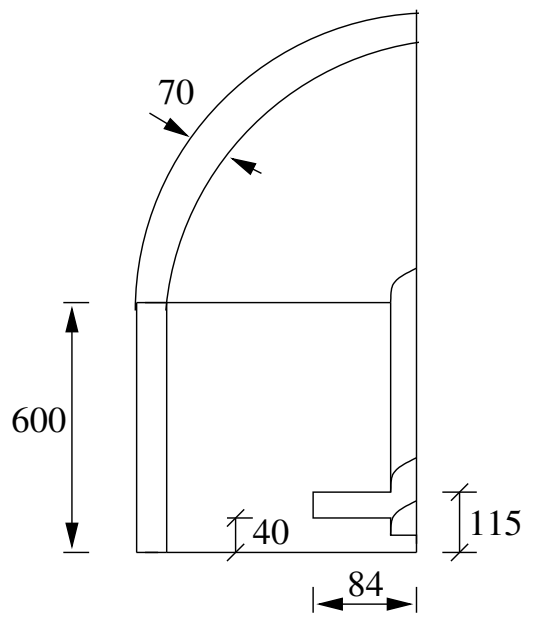

Fig. 24 Test-setup of the Pull out test

The crack speed is shown in Figure 32 and agrees well with computations of other numerical studies, Rabczuk et al. [43]; Belytschko et al. [7]; Xu and Needleman [54]. The crack speed does not exceed the Rayleigh wave speed.

\subsection{Taylor bar impact}

To test the method for multiple cracks with crack junction, we consider a Taylor bar impact. There are experimental results available, see Teng et al. [50]. The Taylor bar has a diameter of $6 \mathrm{~mm}$ and length $30 \mathrm{~mm}$. We consider mod- 


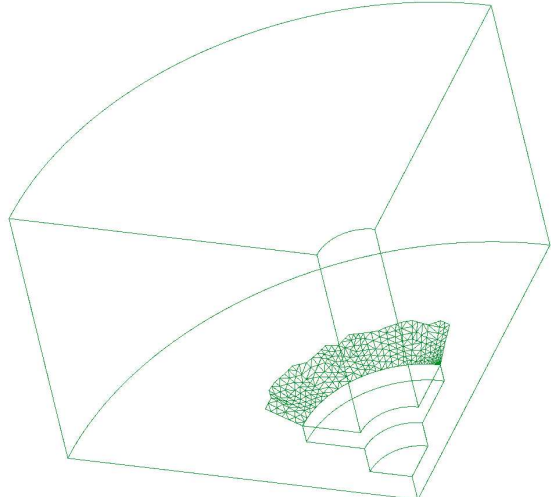

a)

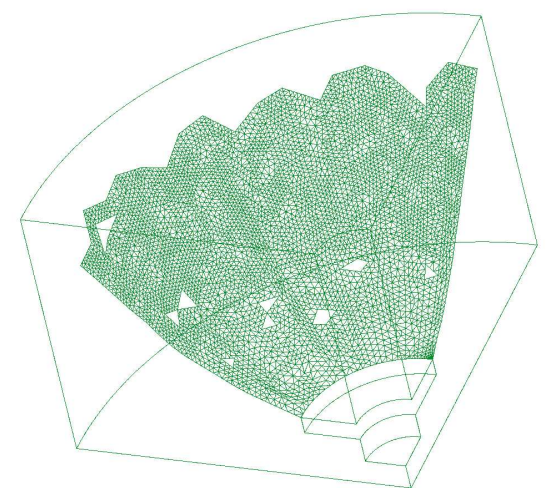

c)

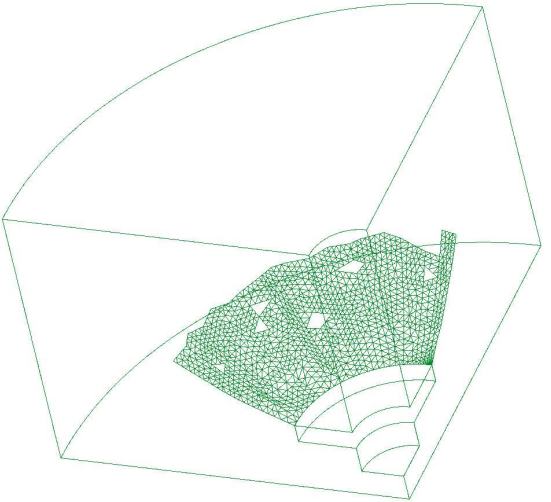

b)

Fig. 25 Crack pattern of the pull-out test

erate impact velocities of $240 \mathrm{~m} / \mathrm{s}$. The material was a $2024-\mathrm{T} 351$ aluminium alloy. We use the Johnson Cook model [25] as in the previous section with Young's modulus $E=74 \mathrm{GPa}$, Poisson ratio $\nu=0.3$, density $0.0027 \mathrm{~g} / \mathrm{mm}^{3}$, a reference strain rate of $3.33 \times 10^{-4}, A=352 \mathrm{MPa}, B=440 \mathrm{MPa}, C=0.0083$, $n=0.42, m=1, c_{v}=875 \mathrm{~J} / \mathrm{kg} \mathrm{C}, T_{r}=296 \mathrm{~K}, T_{m}=775 \mathrm{~K}$ and $\beta=1$. We tested two different discretizations, with approximately 30,000 particles and 70,000 particles. The final deformation of the Taylor bar is shown in Figure 33 for both discretizations. As can be seen, multiple cracking occurs including crack junctions. The results look almost identical and agree well with the results in Teng et al. [50].

\section{CONCLUSIONS AND FUTURE WORK}

This paper presented a local partition of unity enriched 6 EFG method to simulate crack initiation and propagation (including joining cracks) in non-

\footnotetext{
${ }^{6}$ extrinsic enrichment
} 


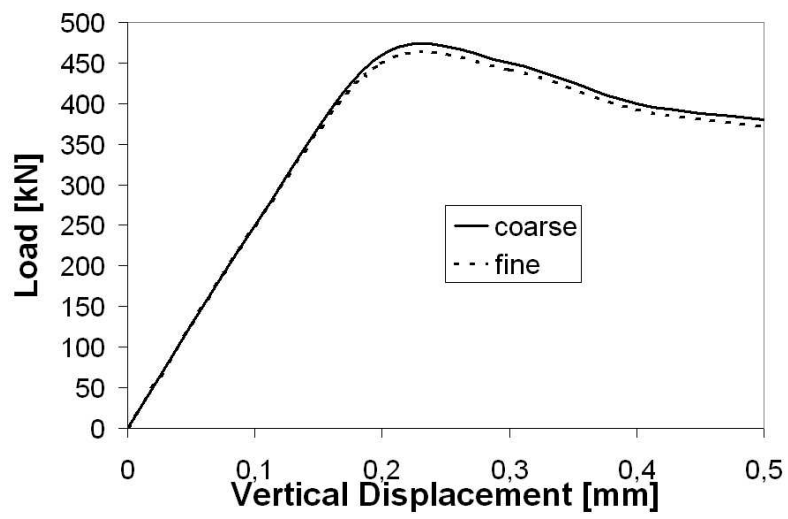

Fig. 26 Load deflection curve of the pull-out test
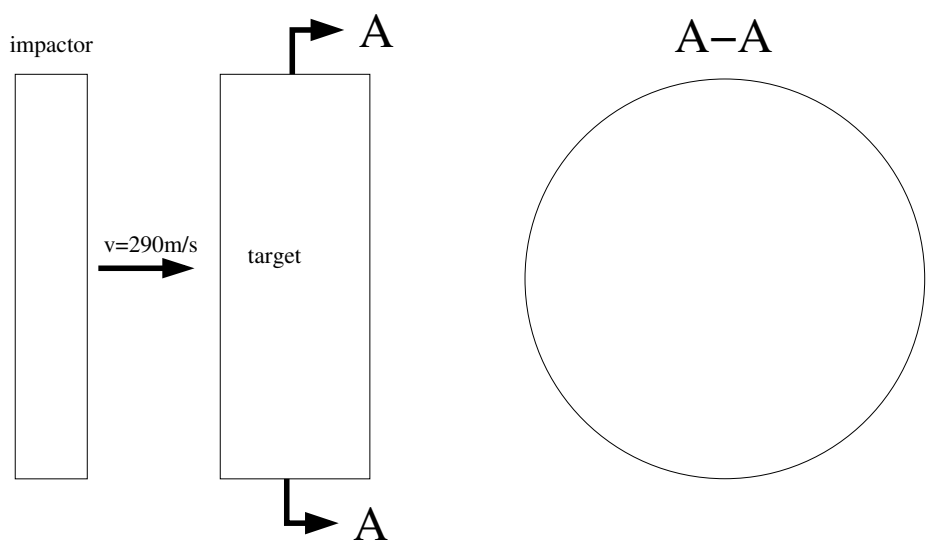

Fig. 27 Schematic test set-up of the flyer plate impact problem

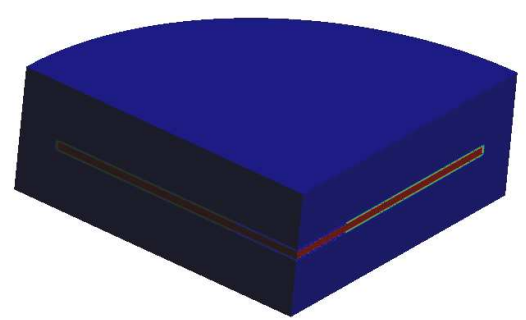

a) $290 \mathrm{~m} / \mathrm{s}$

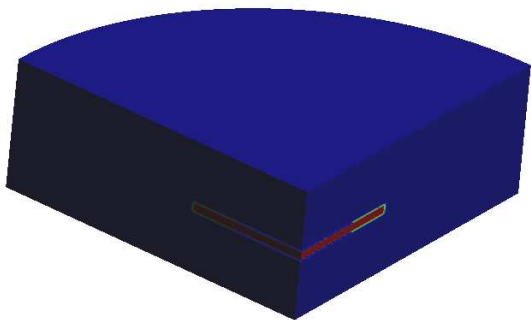

b) $205 \mathrm{~m} / \mathrm{s}$

Fig. 28 One quarter of the impacted plate of the flyer-plate problem for different impact velocities

linear three dimensional solids. The loss of material stability criterion is used 


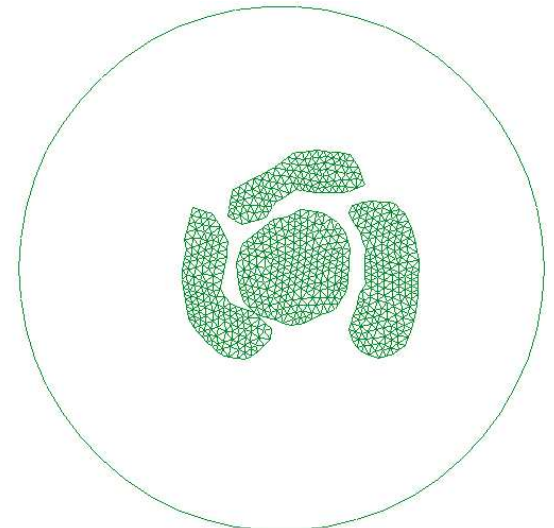

a) $290 \mathrm{~m} / \mathrm{s}$

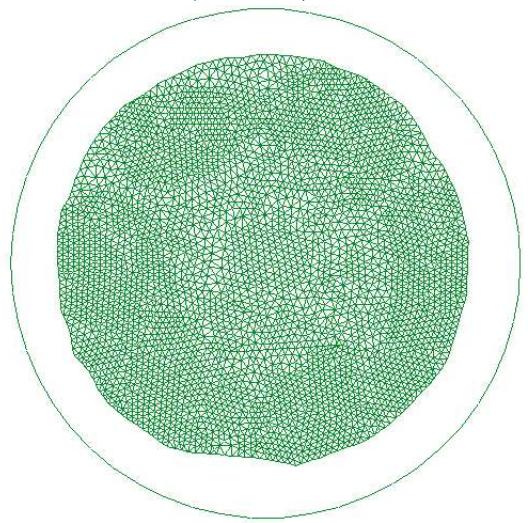

c) $290 \mathrm{~m} / \mathrm{s}$

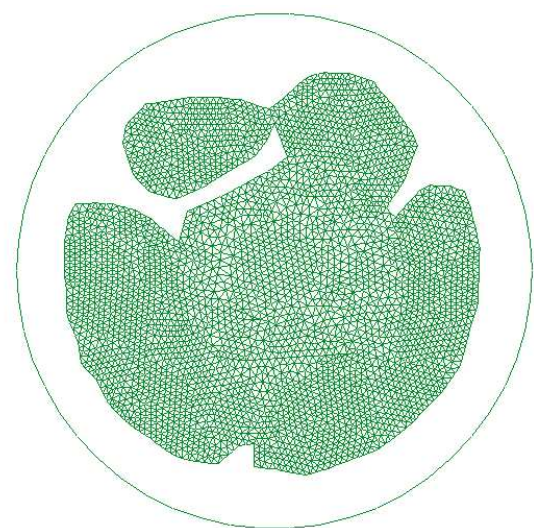

b) $290 \mathrm{~m} / \mathrm{s}$

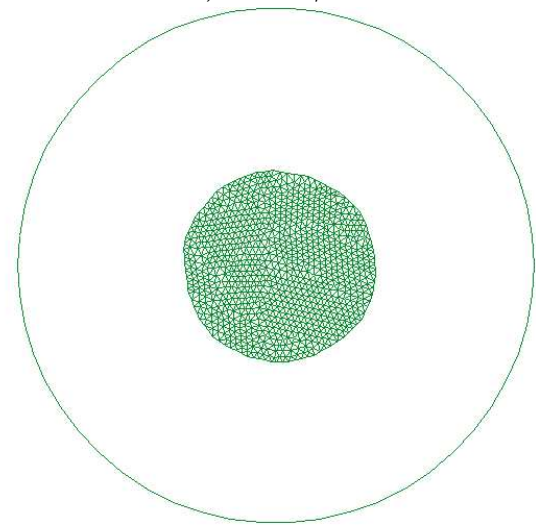

d) $205 \mathrm{~m} / \mathrm{s}$

Fig. 29 Crack pattern of the flyer-plate problem; topview

as transition criterion from the continuum to the discontinuum. The displacement jump is described by additional unknowns and crack front branch enrichment functions are added in the approximation space to close the crack and help represent the asymptotic fields 7 . The crack is discretized by linear triangular elements, and hence, can open only linearly. Adaptivity is employed to improve accuracy.

The most salient feature of the method is that it ensures crack path continuity, thereby avoiding the locking and inaccuracies associated with methods in which crack path continuity is not enforced. The method can handle arbitrary continuum constitutive and cohesive laws. We used the Lemaitre's damage model, a neo Hookean material and the Johnson-Cook plasticity model for the bulk solid and exponential cohesive laws for mixed mode failure. We assure non-penetration of the crack faces by a penalty method.

7 Two near-front enrichment schemes are utilized: the standard Westergaard solution for the purely elastic cases without cohesive zone, and a power law for the cohesive cracks. 


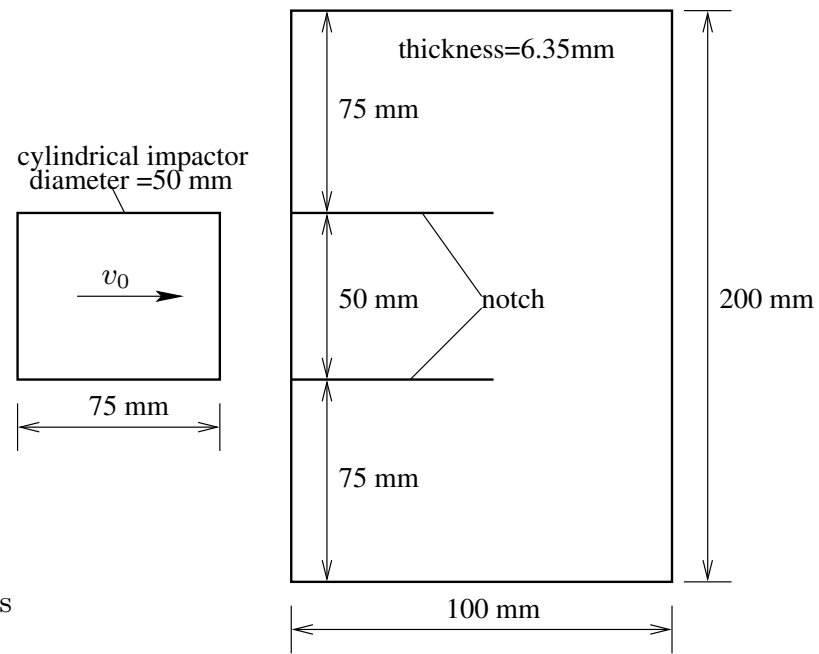

Fig. 30 The Kalthoff problem: test setup

We described in detail the cracking algorithm including crack initiation, crack branching and crack junctions. The most important features of our algorithm are:

1. Cracks may initiate anywhere in the mesh, and we handle complex cases such as those when cracks merge during initiation. Most methods available in the literature do not consider the initiation, but only the propagation of preexisting cracks.

2. We assure crack front continuity, and thereby avoid locking and inaccuracies.

3. We use an adaptive scheme based on the evaluation of the stress gradients to resolve the finest intricacies of the crack surfaces. This is advantageous, since it allows cracks to initiate with a sufficiently small radius, and helps enforcing crack front continuity, through a much better resolution of the crack surface and front.

We applied our method to several static and dynamic problems where experimental data or other numerical results are available. In addition, we tested our method for a penny-shaped mode-I crack problem. In that problem, numerical stress intensity factors were compared to analytical stress intensity factors at different positions along the crack front. The maximum and average error on the stress intensity factor along the front for the coarsest discretization are $0.2 \%$ and $0.085 \%$ respectively.

Our method performed very well for a beam in bending under mixedmode failure and a pull-out test in concrete. Load deflection curves as well as crack patterns agreed with the experimental results and the results did not show mesh-dependency. Using adaptivity, even for very coarse meshes, a high accuracy could be obtained. 


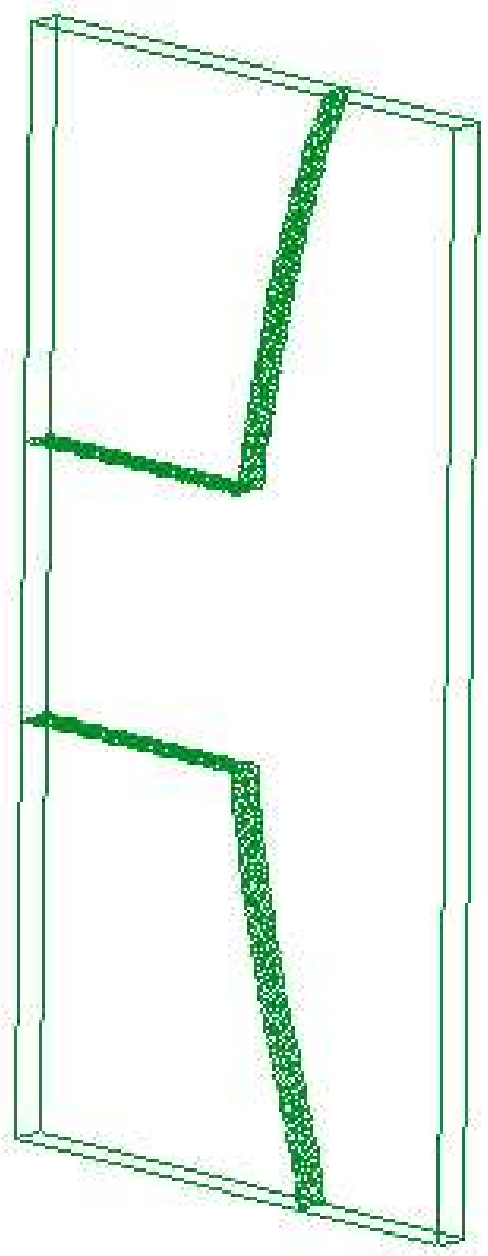

a)

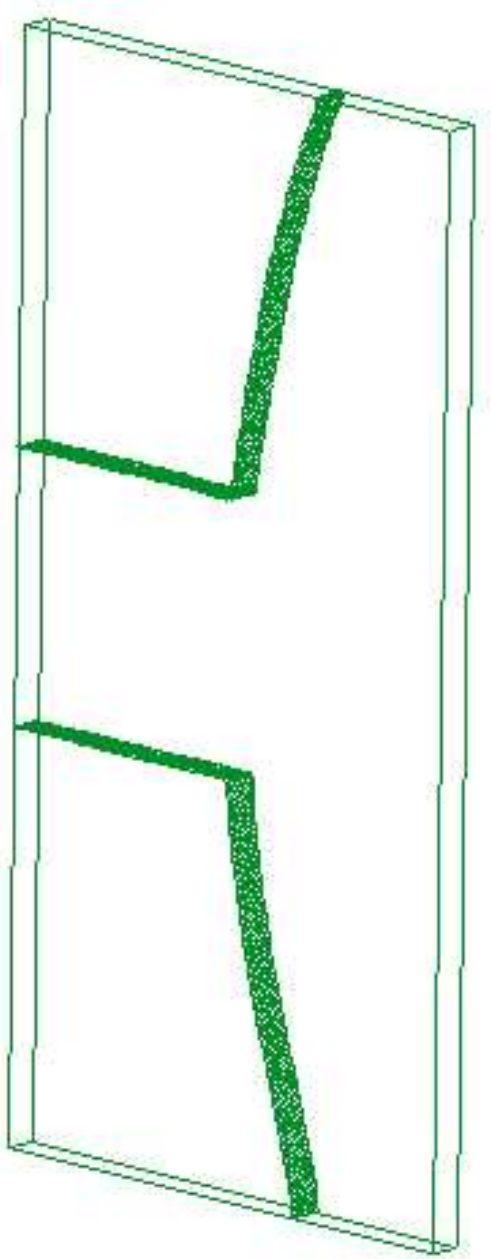

b)

Fig. 31 Crack pattern of the Kalthoff problem

The method was also able to handle dynamic problems with multiple cracking including crack junctions. This was shown by the study of a Taylor bar impact, i.e. a bar shot against a rigid wall with a low-to moderate velocity of $240 \mathrm{~m} / \mathrm{s}$. The crack pattern of the numerical solution agreed well with the experimental results and results of other numerical schemes. For the flyerplate impacts and Kalthoff problem with brittle failure, we obtained equally good results. The spalling plane in the flyer-plate impact simulations agreed well with the experimental results and in the Kalthoff problem, the crack propagation speed was below the Rayleigh wave speed and the different crack speed-time history curves of adaptive and non-adaptive computations agreed very well. Moreover, the crack pattern agreed well with the one of Kalthoff's experiments. 


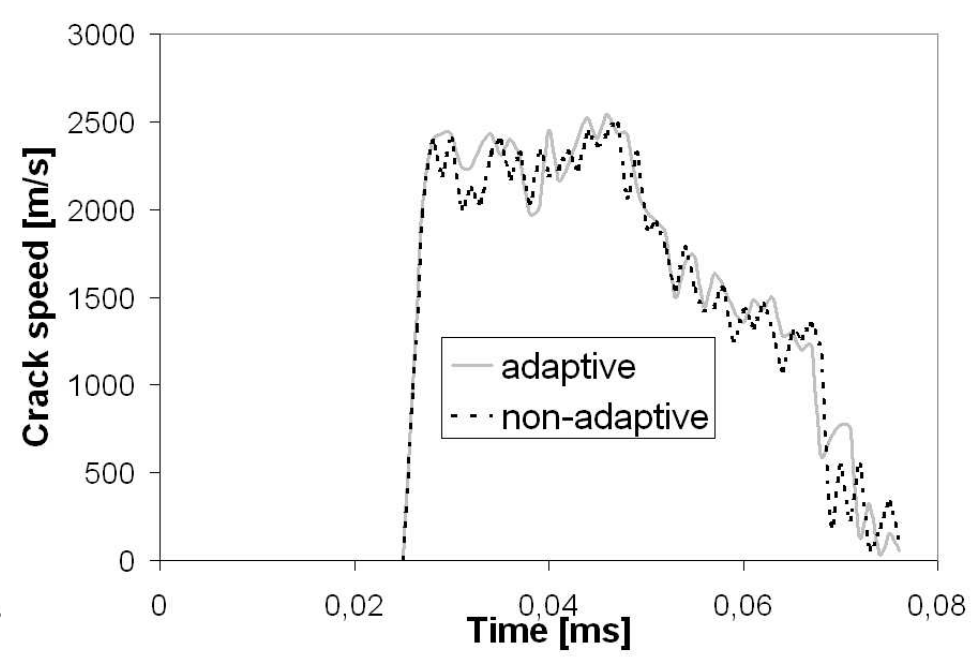

Fig. 32 Crack speed of the Kalthoff problem for different discretizations

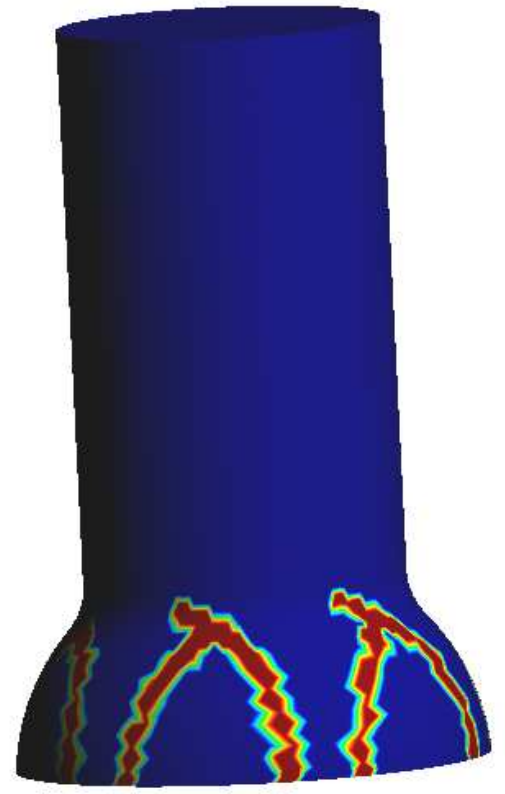

a) 30,000 particles

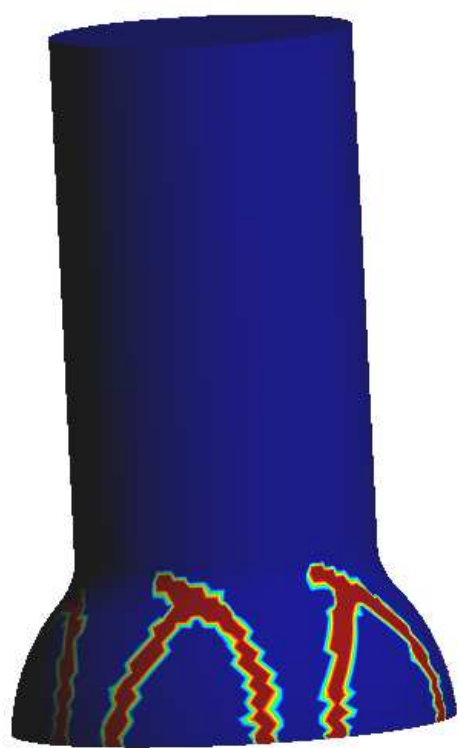

b) 70,000 particles

Fig. 33 Final crack pattern of the Taylor bar problem

There are a few ways in which the proposed method could be improved. For instance it might be advantageous to develop a method where no crack front enrichment is needed. Omitting the crack front enrichment improves 
computational efficiency and removes inherent difficulties when kinks and sharp corners occur at the crack front.

A representation of the crack surface with high-order level sets or NonUniform Rational B-Splines (NURBS) might be advantageous since crack opening can be non-linear as observed in experiments. Of course, in that case, we need a robust integration sheme to track the nonlinear path of the discontinuity.

\section{ACKNOWLEDGEMENT}

The third author thank the support of Korea Institute of Construction and Transportation Technology Evaluation and Planning (KICTTEP) grant 05CTRM-D04-03. 


\section{References}

1. A. Alonso and A. Valli. A domain decomposition approach for heterogenous time-harmonic maxwell equations. Computer Methods in Applied Mechanics and Engineering, 1997.

2. A. Alonso and A. Valli. An optimal domain decomposition preconditioner for low-frequency maxwell equations. Math. Comp., 1999.

3. P.M.A. Areias and T. Belytschko. Analysis of three-dimensional crack initiation and propagation using the extended finite element method. International Journal for Numerical Methods in Engineering, 63:760-788, 2005.

4. M. Arrea and A.R. Ingraffea. Mixed-mode crack propagation in mortar and concrete. Technical Report 81-13, Dept. of Struct. Eng., Cornell University Ithaka, 1982.

5. R. Beck, R. Hiptmair, R.H.W. Hoppe, and B. Wohlmuth. Residual based a posteriori error estimators for eddy current computation. Math modelling Numer. Anal., 2000.

6. J. Bellec and J.E. Dolbow. A note on enrichment functions for modelling crack nucleation. Communications in Numerical Methods for Engineering, 2003.

7. T. Belytschko, H. Chen, J. Xu, and G. Zi. Dynamic crack propagation based on loss of hyperbolicity and a new discontinuous enrichment. International Journal for Numerical Methods in Engineering, 58(12):1873-1905, 2003.

8. T. Belytschko, Y.Y. Lu, and L. Gu. Element-free galerkin methods. International Journal for Numerical Methods in Engineering, 37:229-256, 1994.

9. T. Belytschko, N. Moes, S. Usui, and C. Parimi. Arbitrary discontinuities in finite elements. International Journal for Numerical Methods in Engineering, 50(4):993-1013, 2001.

10. S. Bordas. Extended Finite Element and level set methods with applications to growth of cracks and biofilms. Phd thesis, Northwestern University, December 2003.

11. S. Bordas and B. Moran. Extended finite element and level set method for damage tolerance assessment of complex structures: an object-oriented approach. EFM, 2006. in press.

12. Stéphane Bordas and Antoine Legay. Enriched finite element short course: class notes. In The extended finite element method, a new approach to numerical analysis in mechanics: course notes. Organized by S. Bordas and A. Legay through the EPFL school of continuing education, Lausanne, Switzerland, 12 2005.

13. J. Cervenka. Discrete crack modeling in concrete structures. Phd thesis, University of Colorado, 1994.

14. P. Chevrier and J.R. Klepaczko. Spall fracture: Mechanical and microstructural aspects. Engineering Fracture Mechanics, 1999.

15. D.L. Chopp and N. Sukumar. Fatigue crack propagation of multiple coplanar cracks with the coupled extended finite element/fast marching method. International Journal for Engineering Science, 2003.

16. C. Daux, N. Moes, J. Dolbow, N. Sukumar, and T. Belytschko. Arbitrary branched and intersection cracks with the extended finite element method. International Journal for Numerical Methods in Engineering, 48:1731-1760, 2000 .

17. L. Demkovicz and L. Vardapetyan. Modeling of electromagnetic absorption/scattering problems using hp-adaptive finite elements. Computer Methods in Applied Mechanics and Engineering, 1998.

18. P. Devloo, T.J. Oden, and P. Pattani. An h-p adaptive finite element method for the numerical simulation of compressible flow. Computer Methods in Applied Mechanics and Engineering, 70(2):203-235, 1988.

19. M. Duflot. A meshless method with enriched weight functions for threedimensional crack propagation. International Journal for Numerical Methods in Engineering, 65(12):1970-2006, 2006.

20. R. Galdos. A finite element technique to simulate the stable shape evolution of planar cracks with an application to a semi-elliptical surface crack in a bimate- 
rial finite solid. International Journal for Numerical Methods in Engineering, 40:905-917, 1997.

21. T.C. Gasser and G.A. Holzapfel. Modeling 3d crack propagation in unreinforced concrete using pufem. Computer Methods in Applied Mechanics and Engineering, 2005.

22. A. Gravouil, N. Moes, and T. Belytschko. Non-planar 3D crack growth by the extended finite element and level sets - part ii: Level set update. International Journal for Numerical Methods in Engineering, 53:2569-2586, 2002.

23. R. Hiptmair. Multigrid method for maxwell's equations. SIAM J. Numer. Anal., 1998.

24. P. Houston, H. Perugia, and D. Schötzau. Energy norm a posteriori error estimation for mixed discontinuous galerkin approximations of the maxwell operator. Computer Methods in Applied Mechanics and Engineering, 2005.

25. G.R. Johnson and W.H. Cook. A constitutive model and data for metals subjected to large strains, high strain rates, and high temperatures. In Proc. 7th International Symp. on Ballistics, 1983.

26. J. F. Kalthoff and S. Winkler. Failure mode transition at high rates of shear loading. International Conference on Impact Loading and Dynamic Behavior of Materials, 1:185-195, 1987.

27. P. Krysl and T. Belytschko. The element free galerkin method for dynamic propagation of arbitrary 3-D cracks. International Journal for Numerical Methods in Engineering, 44(6):767-800, 1999.

28. Vardapetyan L. and L. Demkovicz. hp-adaptive finite elements in electromagnetics. Computer Methods in Applied Mechanics and Engineering, 1999.

29. J. Lemaitre. Evaluation of dissipation and damage in metal submitted to dynamic loading. Proceedings ICM 1, 1971.

30. S. Li and Bo C. Simonson. Meshfree simulation of ductile crack propagation. International Journal of Computational Methods in Engineering Science and Mechanics, 6:1-19, 2004.

31. W.K. Liu, S. Jun, S. Li, J. Adee, and T. Belytschko. Reproducing kernel particle method for structural dynamics. International Journal for Numerical Methods in Engineering, 38:1665-1679, 1995.

32. W.K. Liu, S. Li, and T. Belytschko. Moving least square reproducing kernel method. (i) methodology and convergence. Computer Methods in Applied Mechanics and Engineering, 143:113-154, 1997.

33. Y. Liu, S. Murakami, and Y. Kanagawa. Mesh-dependence and stress singularity in finite element analysis of creep crack growth by continuum damage mechanics approach. European Journal of Mechanics A/Solids, 13:395-417, 1994.

34. S.H. Lo, C.Y. Dong, and Y.K. Cheung. Integral equation approach for 3d multiple crack problems. Engineering Fracture Mechanics, 2005.

35. R. Loehner. Applied CFD Techniques: An introduction based on Finite Element Methods. John Wiley and Sons, LTD, 2001.

36. L.F. Martha, P.A. Wawrzynek, and A.R. Ingraffea. Arbitrary crack representation using solid modeling. Engng. Computers, 9:63-82, 1993.

37. N. Moes, J. Dolbow, and T. Belytschko. A finite element method for crack growth without remeshing. International Journal for Numerical Methods in Engineering, 46(1):133-150, 1999.

38. N. Moes, A. Gravouil, and T. Belytschko. Non-planar 3-D crack growth by the extended finite element method and level sets, parti: Mechanical model. International Journal for Numerical Methods in Engineering, 53(11):2549-2568, 2002.

39. P. Monk. On the p- and hp-extension of nedelec's curl-conforming elements. Journal of Computational and Applied Mathematics, 1994.

40. P. Monk. A posteriori error indicators for maxwell's equations. Journal of Computational and Applied Mathematics, 1998.

41. R. W. Ogden. Non-linear elastic deformations. Halsted Press, New York, 1984

42. J. Oliver, A.E. Huespe, and P.J. Snchez. A comparative study on finite elements for capturing strong discontinuities: E-fem vs. x-fem. Computer Methods in 
Applied Mechanics and Engineering, in press, 2006.

43. T. Rabczuk, , P.M.A. Areias, and T. Belytschko. A simplified meshfree method for shear bands with cohesive surfaces. International Journal for Numerical Methods in Engineering, submitted.

44. T. Rabczuk and T. Belytschko. Adaptivity for structured meshfree particle methods in 2D and 3D. International Journal for Numerical Methods in Engineering, 63(11):1559-1582, 2005.

45. T. Rabczuk, T. Belytschko, and S.P. Xiao. Stable particle methods based on lagrangian kernels. Computer Methods in Applied Mechanics and Engineering, 193:1035-1063, 2004

46. W. Rachowicz and L. Demkovicz. An hp-adaptive finite element method for electromagnetics-part i: data structure and constrained approximation. Computer Methods in Applied Mechanics and Engineering, 2000.

47. W. Rachowicz and L. Demkovicz. An hp-adaptive finite element method for electromagnetics-part ii: a $3 \mathrm{~d}$ implementation. International Journal for $\mathrm{Nu}$ merical Methods in Engineering, 2002.

48. D.C. Jr. Simkins and S. Li. Meshfree simulations of ductile failure under thermal-mechanical loads. Computational Mechanics, 2006.

49. N. Sukumar, B. Moran, T. Black, and T. Belytschko. An element-free galerkin method for three-dimensional fracture mechanics. Computational Mechanics, 20:170-175, 1997.

50. X. Teng, T. Wierzbicki, S. Hiermaier, and I. Rohr. Numerical prediction of fracture in the taylor test. International Journal of Solids and Structures, 2005.

51. G. Ventura, J. Xu, and T. Belytschko. A vector level set method and new discontinuity approximations for crack growth by efg. International Journal for Numerical Methods in Engineering, 54(6):923-944, 2002.

52. G. Xu, F.P. Bower, and M. Ortiz. An analysis of non-planar crack growth under mized mode loading. International Journal of Solids and Structures, 31: 2167-2193, 1994.

53. G. Xu and M. Ortiz. A variational boundary integral method for the analysis of $3 \mathrm{~d}$ cracks of arbitrary geometry modellled as continuous distributions of dislocation loops. International Journal for Numerical Methods in Engineering, 36:3675-3701, 1993.

54. X.-P. Xu and A. Needleman. Numerical simulations of fast crack growth in brittle solids. Journal of the Mechanics and Physics of Solids, 42:1397-1434, 1994.

55. G. Zi, J.-H. Song, E. Budyn, S.-H. Lee, and T. Belytschko. A method for grawing multiple cracks without remeshing and its application to fatigue crack growth. Modelling and Simulation in Materials Science and Engineering, 12 (1):901-915, 2004.

56. O.C. Zienkiewicz and J.Z. Zhu. The superconvergent patch recovery and a posteriori error estimates. Part I: the recovery technique. International Journal for Numerical Methods in Engineering, 33:1331-1364, 1992. 